\title{
TRiM: Enhancing Processor-Memory Interfaces with Scalable Tensor Reduction in Memory
}

\author{
Jaehyun Park ${ }^{*}$ \\ Seoul National University \\ Seoul, South Korea \\ jhpark@scale.snu.ac.kr \\ Eojin Lee \\ Inha University \\ Incheon, South Korea \\ ejlee@inha.ac.kr
}

\author{
Byeongho Kim* \\ Seoul National University \\ Seoul, South Korea \\ bhkim@scale.snu.ac.kr \\ Minsoo Rhu \\ KAIST \\ Daejeon, South Korea \\ minsoo.rhu@gmail.com
}

\author{
Sungmin Yun \\ Seoul National University \\ Seoul, South Korea \\ sungmin.yun@scale.snu.ac.kr \\ Jung Ho Ahn \\ Seoul National University \\ Seoul, South Korea \\ geniajh@gmail.com
}

\begin{abstract}
Personalized recommendation systems are gaining significant traction due to their industrial importance. An important building block of recommendation systems consists of the embedding layers, which exhibit a highly memory-intensive characteristic. A fundamental primitive of embedding layers is the embedding vector gathers followed by vector reductions, exhibiting low arithmetic intensity and becoming bottlenecked by the memory throughput. To tackle such a challenge, recent proposals employ a near-data processing (NDP) solution at the DRAM rank-level, achieving impressive performance speedups. We observe that prior rank-levelparallelism-based NDP solutions leave significant performance potential on the table as they do not fully reap the abundant transfer throughput inherent in DRAM datapaths.

We propose TRiM, an NDP architecture for accelerating recommendation systems. Based on the observation that the DRAM datapath has a hierarchical tree structure, TRiM augments the DRAM datapath with "in-DRAM" reduction units at the DDR4/5 rank/bankgroup/bank level. We modify the interface of DRAM to provide commands effectively to multiple reduction units running in parallel. We also propose a host-side architecture with hot embedding-vector replication to alleviate the load imbalance that arises across the reduction units. An optimal TRiM design based on DDR5 achieves up to a $7.7 \times$ and $3.9 \times$ speedup and reduces by $55 \%$ and $50 \%$ the energy consumption of the embedding vector gather and reduction over the baseline and the state-of-the-art NDP architecture with minimal area overhead equivalent to $2.66 \%$ of DRAM chips.
\end{abstract}

\section{CCS CONCEPTS}

- Computer systems organization $\rightarrow$ Parallel architectures.

${ }^{*}$ Both authors contributed equally to the paper.

This work is licensed under a Creative Commons Attribution International 4.0 License.

MICRO '21, October 18-22, 2021, Virtual Event, Greece

(C) 2021 Copyright held by the owner/author(s)

ACM ISBN 978-1-4503-8557-2/21/10.

https://doi.org/10.1145/3466752.3480080

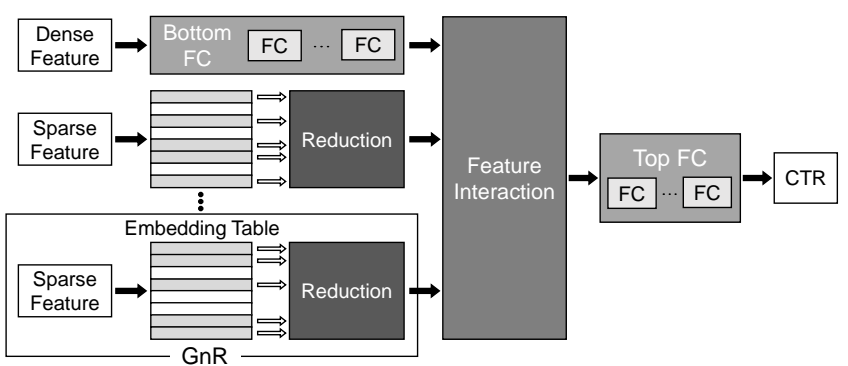

Figure 1: Simplified architecture of a RecSys model. We mainly target accelerating GnR (gather-and-reduction), which gathers embedding vectors from embedding tables and reduces them to produce one vector per table. FC and CTR stand for fully connected layer and click-through-rate.

\section{KEYWORDS}

Memory system, near-data processing, DRAM, main memory

ACM Reference Format:

Jaehyun Park, Byeongho Kim, Sungmin Yun, Eojin Lee, Minsoo Rhu, and Jung Ho Ahn. 2021. TRiM: Enhancing Processor-Memory Interfaces with Scalable Tensor Reduction in Memory. In MICRO-54: 54th Annual IEEE/ACM International Symposium on Microarchitecture (MICRO '21), October 18-22, 2021, Virtual Event, Greece. ACM, New York, NY, USA, 14 pages. https: //doi.org/10.1145/3466752.3480080

\section{INTRODUCTION}

Personalized recommendation systems aim to provide the content preferred by users based on their experience. Companies such as Facebook [20], YouTube [8], and Alibaba [60] are applying deep learning to their recommendation systems to maximize the accuracy of selecting user-preferred content. Recommendation systems based on deep learning (RecSys) have recently gained significant attention within the research community due to their industrial importance. For instance, Facebook states that recommendation systems (e.g., the Deep Learning Recommendation Model (DLRM) [20]) account for $80 \%$ of the AI inference cycles in their datacenters.

A RecSys model utilizes the features of the target user and item to predict the click-through-rate (CTR), which indicates the probability of the user clicking the item (Figure 1). The input features consist of both sparse, categorical features and dense, continuous features. A 
dense feature is a vector whose elements are floating-point numbers. The vector is passed through bottom fully-connected (FC) layers and is collectively translated to one intermediate vector. A sparse feature is represented as a sequence of indices. A number of vectors are fetched from an embedding table using the indices of a sparse feature, which are subsequently reduced to one intermediate vector using element-wise operations (henceforth referred to as tensor gather-and-reduction $(\mathrm{GnR}))$. These intermediate vectors produced by $\mathrm{GnR}$ operations are combined through feature interaction, which is conducted by a batched matrix multiplication, and passed through top FC layers to produce a CTR.

Prior work [20] has shown that both FC layers and GnR take up a significant fraction of the end-to-end inference time of RecSys. While there have been numerous studies focusing on accelerating FC layers, only a handful of prior works have explored the acceleration of GnR, whose characteristics significantly differ from those of FC layers.

Because the embedding table used in RecSys requires a large memory capacity, the embedding table must be stored in main memory. The performance of the GnR operation is constrained by the main-memory bandwidth as the vectors for the GnR operation are fetched from main memory. Increasing the memory bandwidth in a memory channel accompanies an increase in the number of pins or the frequency of the pins, both of which are highly costly. Consequently, TensorDIMM [35] and RecNMP [29] are two recent studies that explored the efficacy of near-data processing (NDP) for accelerating GnR. These architectures accelerate the GnR operation by placing processing elements (PEs) dedicated to each rank (adopting rank-level parallelism) in the buffer chip of DRAM, thereby utilizing the internal bandwidth which is equal to the channel bandwidth multiplied by the number of ranks in the channel. However, we observe that rank-level parallelism does not fully reap the maximum potential of NDP acceleration, leaving significant performance left on the table.

We propose an NDP-based GnR accelerator called the TRiM (Tensor Reduction in Memory) architecture, which is based on DDR4/5 DRAM. Our key approach is to utilize the hierarchical, tree topology of the DRAM datapath structure [10] to seamlessly extract additional internal bandwidth compared to rank-level-parallelismbased NDPs, improving the GnR performance. Compared to prior approaches, a key contribution of our study is the identification of effective embedding table mapping schemes when using multiple ranks/bank-groups/banks. In our work, we compare the internal bandwidth utilization and energy efficiency in various mapping schemes. As with the existing DRAM interface, if command/address (C/A) signals are transferred only through the C/A path, we cannot fully utilize all PEs placed in/near the main-memory architecture. Therefore, we propose and analyze multiple C/A transfer schemes that can amplify the C/A signal bandwidth without significantly modifying the conventional DRAM interface. Among the embodiments of TRiM architectures, we find the optimal design that can effectively process $\mathrm{GnR}$ according to the workload characteristics.

Another important contribution is a detailed analysis of the loadimbalance issue that occurs when multiple ranks/bank-groups/banks process different numbers of lookups per GnR operation, a key limitation of NDP architectures including our design and RecNMP. To address this problem, we propose hot-entry replication by utilizing the workload characteristic according to which the number of accesses is skewed to a small fraction of entries in the embedding table in RecSys. This scheme improves load balancing without any additional modifications to the DRAM interface. We also develop a novel data reliability solution for TRiM architectures that cannot exploit the conventional rank-level error correction code (ECC). Because $\mathrm{GnR}$ accesses the embedding tables in a read-only manner, we repurpose the existing on-die ECC [26] to only detect but not correct errors during GnR.

In this paper, we make the following key contributions:

- We conduct a quantitative analysis of existing NDP solutions aimed at accelerating the GnR operation.

- We propose TRiM, an NDP architecture that accelerates the GnR operation by utilizing the features of the DRAM data/control path structure without causing significant changes in the conventional DRAM interface.

- We propose hot-entry replication utilizing the characteristics of the RecSys workload to alleviate the load imbalance problem that occurs in TRiM.

- The optimized TRiM architecture based on DDR4/5 DRAM improved the performance of $\mathrm{GnR}$ by up to $7.7 \times$ and $3.9 \times$, respectively, compared to the baseline and state-of-the-art architectures.

\section{BACKGROUND AND MOTIVATION}

\subsection{Tensor Gather-and-Reduction in RecSys}

Tensor gather-and-reduction (GnR) performs a simple reduction operation (e.g., an element-wise sum for SparseLengthsSum (SLS) in Caffe2 [1]) of embedding vectors collected from multiple embedding table lookups. In DLRM [46], a representative RecSys model, one GnR operation performs generally between 20 and 80 lookups. An embedding table takes the form of a matrix (rank-2 tensor) in which each row holds one embedding vector (rank-1 tensor). The number of elements in a row (hereafter referred to as the vector length, $v_{l e n}$ ) typically ranges from 32 to 256 [54]. The on-chip storage of a processor is too small to store all of the embedding tables of RecSys, the size of which can exceed hundreds of GBs. Therefore, embedding vectors are mostly read from the main-memory DRAM.

As the compute to memory access ratio of $\mathrm{GnR}$ is extremely low with little locality (i.e., several KBs to MBs of DRAM reads over several tens to hundreds of GBs of embedding tables), GnR is highly memory intensive. This property renders $\mathrm{GnR}$ a prime candidate for acceleration using near-data processing (NDP $[2,6,14])$ at the processor-memory interface. The NDP architecture places processing elements (PEs) near the DRAM datapath I/O; thus, data read from the DRAM chip are processed within or near the DRAM chip. By fully utilizing the characteristics of the DRAM data/control path, the NDP architecture can achieve high performance.

\subsection{DRAM Data/Control Path Architecture}

The datapath of popular main-memory systems such as DDR4/5 DRAM [24, 26] takes a hierarchical tree structure (see Figure 2(a)), i.e., a memory channel as a root node (depth-0) consisting of a primary host memory controller (MC) and multiple, secondary DRAM ranks (depth-1) connected through a depth-1 data bus. Each 


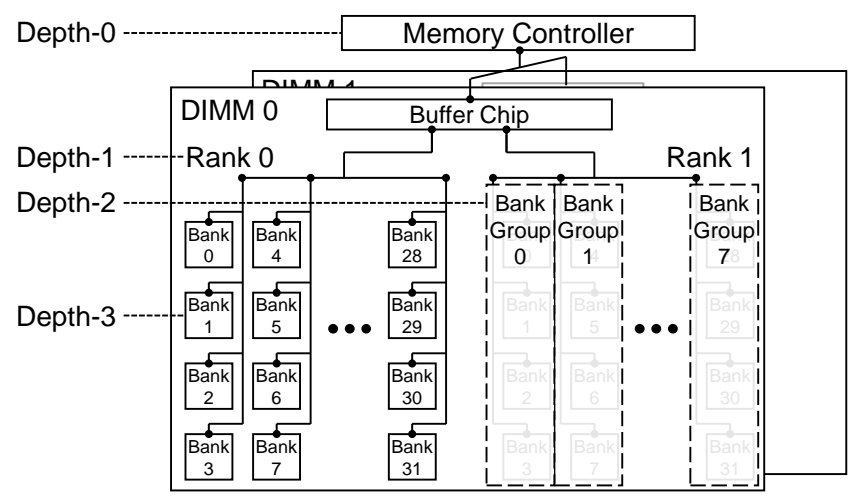

(a)

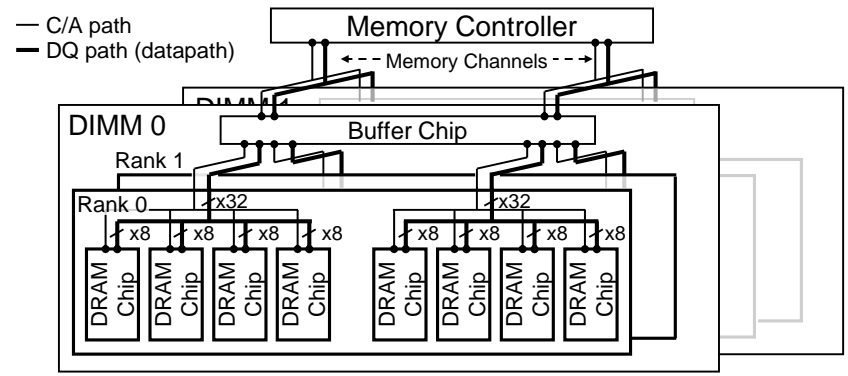

(b)

Figure 2: (a) Simplified DRAM datapath assuming a single memory channel and (b) module organization of DDR5 DRAM with 2 DIMMs $\times 2$ ranks connecting two memory channels.

rank consists of several DRAM chips, all receiving the same command/address information by broadcasting and transferring the corresponding data accordingly. The datapath entering the rank is physically divided and connected to each DRAM chip (see Figure 2(b)). To maximize memory-level parallelism, several ranks can physically be housed within a DIMM module.

In DDR5 DRAM, a rank consists of eight bank-groups (depth2), each packed with four banks (depth-3). Similar to the depth-1 data bus, the depth-2 data bus and the depth-3 data bus are shared between one rank and eight bank-groups, and between one bankgroup and four banks, respectively. All of the banks in a DRAM chip operate independently, albeit only one bank can occupy depth-1/2/3 data buses at any given time, and the frequency of row activations (the process of moving a row of a DRAM bank to the sense amplifiers in the bank preparing for reading or writing the data) is limited (e.g., due to tRRD and tFAW) such that it cannot exceed the power limit of the DRAM chip.

The notion of a bank-group did not exist until DDR4. DDR4 DRAM started adopting such a concept to retain the frequency of DRAM banks at a low level while increasing the data transfer rate and hence the bandwidth. Because the frequency inside a bank-group bus is lower than that outside a bank-group, the consecutive read delay within a bank-group (tCCD_L) is greater than that between the bank-groups (tCCD_S). The bank-group organizes a level of the hierarchy between the ranks and banks, connecting hierarchical multi-drop buses among them.

DDR5 DRAM allows one DIMM to be connected to two channels, giving it a physically different configuration from previous DDR generations where one DIMM is connected to one channel. However, as the two channels operate independently, it can be seen that they have separate data/control paths.

The control path of the main-memory systems is structured similarly. An MC sends command and address (C/A) signals to all connected DRAM ranks through a C/A bus in a broadcast manner. Each rank drops the signals if it is not the right destination. A buffer chip or chipset connects an MC and the ranks in a module to alleviate signal integrity issues [25]. We assume a data/control path dedicated to each rank in the buffer chip as in MCN DIMM [4], which is also assumed in the previous studies [29, 35] (Figure 2(b)).

Because the datapath of DRAM has a hierarchical (tree-like) bus structure, the bus between the $\mathrm{MC}$ and the rank can only be utilized by one rank at a time. However, if a processing unit for $\mathrm{GnR}$ is placed within the buffer chip, the datapath between the MC and the buffer chip is not utilized during GnR. Therefore, all ranks can simultaneously transfer data to the corresponding processing units in the connected buffer chips. We exploit this characteristic to accelerate $\mathrm{GnR}$ with a higher internal bandwidth.

\section{ANALYZING PRIOR NDP ACCELERATORS FOR GNR}

\subsection{TensorDIMM and RecNMP}

Figure 3 explains how the baseline system (Base) and prior NDP accelerators handle GnR. In Base using a conventional module-based DDR5 DRAM, only one rank can occupy the bus and transfer data in a channel at a time because all ranks in the channel share the depth1 bus. TensorDIMM [35] and RecNMP [29], two recently proposed NDP accelerators for GnR, employ one processing element (PE) per rank, locating them inside the buffer chips. Each PE performs GnR for (a portion of) an embedding vector in parallel. As the depth-1 bus is not utilized during $\mathrm{GnR}$ in each module, each PE in a rank can receive data independently. Therefore, PEs in different ranks can receive data in parallel, and the aggregated bandwidth for GnR can be as high as the channel bandwidth multiplied by the number of ranks in a channel (rank-level parallelism). Furthermore, as the embedding vectors for $\mathrm{GnR}$ are transferred to the buffer chips but not through the depth-1 bus, data transfer energy is saved. Only the reduced vectors are transferred through the depth-1 bus.

A key factor that differentiates Base from prior NDP accelerators for $\mathrm{GnR}$ is the manner in which the embedding tables are mapped across the memory subsystem. In Base, the elements in an embedding vector exist at consecutive addresses in a single DRAM row. Therefore, the embedding vector elements are sequentially transferred to the host MC after activating the DRAM row that contains the target vector.

TensorDIMM and RecNMP adopt different embedding table mapping and reduction strategies. TensorDIMM splits the embedding table vertically so that each partition only has a portion of the embedding vector, each of which is mapped to different ranks (vertical partitioning, vP). Because the $\mathrm{PE}$ dedicated to each rank produces a portion of a reduced vector from $\mathrm{GnR}$, the reduced vector in each PE 
$\square$ Processing Element (PE) for Reduction $\quad$ mactivate (ACT) $\square$ Data Transfer RA: Rank BG: Bank Group

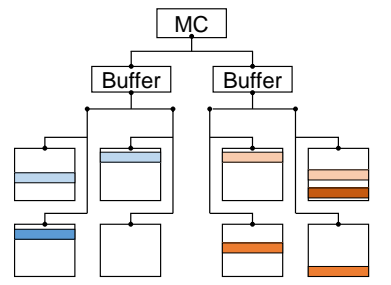

(a) Baseline system (Base)

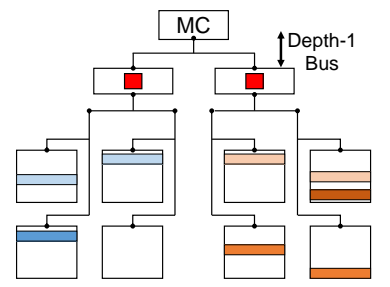

(c) NDP architecture adopting horizontal partitioning (HOR)
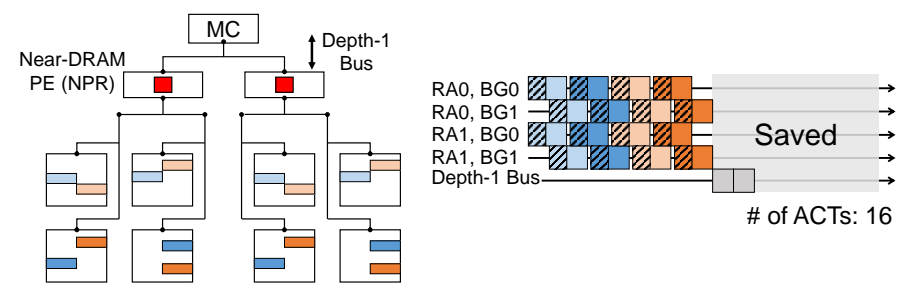

(b) NDP architecture adopting vertical partitioning (VER)

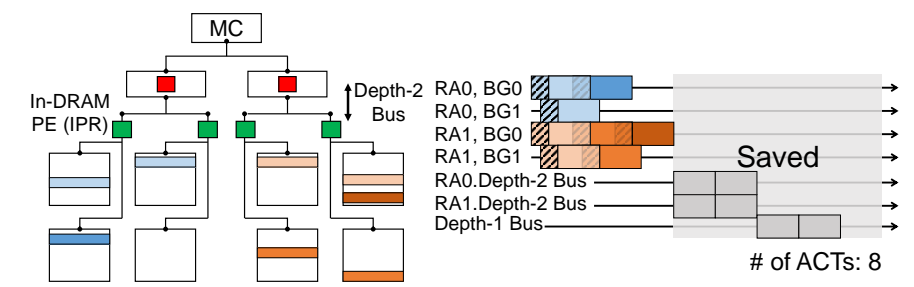

(d) TRiM-G (an example of the proposed architecture)

Figure 3: Exemplar GnR in the baseline (Base) and the evaluated NDP architectures. Each diagram on the left simplifies conceptual DRAM modules, where each has one DRAM rank consisting of two bank-groups, each packed with two banks, showing how embedding vectors are mapped to the DRAM chips. The timing diagram on the right shows the states of bank-groups and buses while transferring data or conducting partial GnR near/in DRAM.

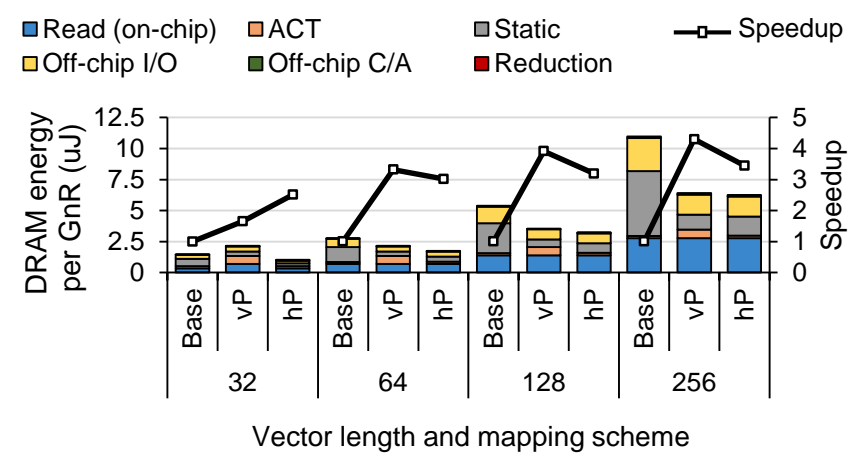

Figure 4: Speedup and DRAM energy breakdown of Base and the state-of-the-art NDP accelerators when performing $\mathrm{GnR}$. The evaluation assumes a DDR5-4800 DRAM module with four ranks (Section 5 details the workloads and the configuration for both the Base and NDP architectures).

is concatenated with the other portions at the host. In contrast, RecNMP evenly distributes the entries of an embedding table to each rank (horizontal partitioning, $\mathrm{hP}$ ). After performing the reduction of vectors in the PEs, each PE sends a partial sum of the vectors to the host, and the host reduces the partial sums. Because the vector is reduced by an element-wise operation, the elements that arrive at the buffer chip first can be reduced and transferred to the host before the entire vector arrives at the buffer chip. Moreover, data transfer to the host can be overlapped by the subsequent reduction of vectors by another GnR in the PEs.

While the performance benefits of employing NDP for GnR acceleration are clear, we observe several important design overheads that the prior works do not address appropriately. An NDP architecture adopting $\mathrm{vP}$ (VER) distributes one vector to multiple ranks evenly such that the number of row activations (ACTs) for a GnR operation becomes proportionally larger as a function of how many ranks are used to (vertically) partition the embedding table (e.g., Figure $3(\mathrm{~b})$ incurs $2 \times$ more ACTs compared to Figure $3(a, c)$ ). Consequently, a key limitation of VER is that it consumes significantly more energy in ACTs for GnR than Base and HOR (NDP architecture adopting $\mathrm{hP}$ ). Moreover, if the partitioned vector size in VER is smaller than the DRAM access size, the internal data read/write bandwidth is wasted due to redundant data reads.

With regard to the hP strategy, HOR must transfer different C/A signals to each rank because each PE processes embedding lookups of different entries. Therefore, HOR requires a higher C/A bandwidth than VER, which broadcasts $\mathrm{C} / \mathrm{A}$ signals to all ranks that process the different elements of the same embedding vector. RecNMP mitigates this high C/A bandwidth pressure by compressing a pair of activate/precharge (ACT/PRE) commands and several read (RD) commands to one custom instruction because all elements in an embedding vector exist in one DRAM row. However, the loadimbalance issue also becomes problematic in HOR as each rank may receive a different number of embedding lookups for GnR.

\subsection{Quantitative Analysis}

To quantify the pros and cons of VER and HOR in GnR, we compare the performance and DRAM energy consumption of VER and HOR against Base without caching recently accessed embeddings when sweeping the values of $v_{l e n}$ from 32 to 256 (see Figure 4 ). The performance of VER and HOR is significantly improved because both can utilize the abundant internal bandwidth of the DRAM. VER shows up to $4.3 \times$ higher performance (similar to the number of ranks per channel, $N_{\text {rank }}$ ) when $v_{\text {len }}$ is 256 , but the achieved speedup from VER becomes only $1.6 \times$ when $v_{l e n}$ is 32 . For a $v_{l e n}$ value of 64, each rank is assigned with 16-length partitioned vector 
elements, which correspond to $64 \mathrm{~B}$, identical to the single DRAM access size. Because there is little spatial locality when accessing the embedding table, vectors are mostly read from different DRAM rows. Therefore, in most cases, a separate instance of row activation is required for every vector read operation within a given rank. However, when $v_{\text {len }}$ is 32 where the partitioned embedding vector size of VER is smaller than the minimum DRAM access granularity of $64 \mathrm{~B}$, half of the DRAM bandwidth is wasted, resulting in the achievement of only half of the performance benefit of $v_{l e n}$ of 64 . HOR overcomes the aforementioned limitation of VER when the GnR's memory access stream can sufficiently utilize the internal bandwidth of all ranks, even when $v_{l e n}$ is 32 . However, the performance of HOR decreases by about $10 \%$ to $20 \%$ compared to VER due to the load-balancing issue.

HOR is more energy-efficient than VER because the ACT energy of VER is four times larger than that of Base and HOR (equivalent to $N_{\text {rank }}$ ). In particular, when $v_{\text {len }}$ is 32 and 64, VER consumes more DRAM energy than Base and HOR because the ACT energy accounts for a large portion of the total DRAM energy consumption As $v_{l e n}$ increases, the ACT energy is amortized over the off-chip I/O energy, the DRAM read energy, and the DRAM static energy. When $v_{\text {len }}$ is 256, off-chip data transfers from a buffer chip to a host MC are significantly reduced in VER and HOR compared to those in Base. Also, the DRAM static energy in NDP architectures is reduced due to the speedup in GnR. Thus, the energy consumption of VER and HOR decreases by $42 \%$ and $43 \%$ over Base, respectively. Owing to the wasted internal bandwidth, as mentioned above, for VER when $v_{l e n}$ is 32 , there is no significant difference in the energy consumption between the two $v_{\text {len }}$ values of 32 and 64 . The C/A signaling and reduction operation slightly affects the total energy consumption.

\subsection{Additional Schemes for Accelerating GnR}

RecNMP applied additional optimizations to improve GnR performance. First, it implements a cache (RankCache) inside the buffer chip to store entries with high access rate. RecNMP improves the GnR performance by taking advantage of the temporal locality of access to the embedding table in RecSys workloads. Second, to alleviate the load imbalance problem that occurs in HOR, RecNMP puts $\mathrm{GnR}$ operations together in a single $\mathrm{GnR}$ batch to process several $\mathrm{GnR}$ operations at a time. When processing a single $\mathrm{GnR}$ operation (when the number of $\mathrm{GnR}$ operations per batch, $N_{G n R}$, is $1)$, the number of lookups $\left(N_{\text {lookup }}\right)$ handled by each rank is highly uneven, resulting in a significant performance drop due to the loadimbalance issue. However, if multiple GnR operations (when $N_{G n R}$ $>1$ ) are processed at once, the total number of lookups in the batch increases, which relieves the load-imbalance problem.

\section{TENSOR REDUCTION IN MEMORY}

Although TensorDIMM and RecNMP achieve decent speedups, we observe that there are substantial untapped, further performance improvement opportunities to be gained by leveraging the internal data transfer bandwidth at the DRAM datapath. Because the datapath of DRAM is organized as a tree structure, if there are PEs dedicated to memory nodes (e.g., ranks, bank-groups, and banks) above a certain depth in an NDP architecture, embedding vectors can be
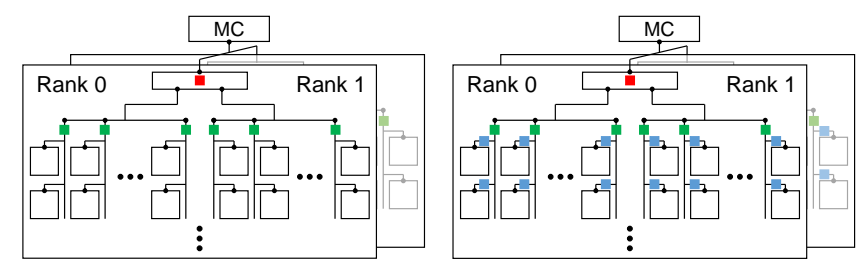

Figure 5: High-level overview of TRiM-G/B (left/right). Red/Green/Blue squares correspondingly denote PE located near the DRAM datapath dedicated per Rank/bankGroup/Bank.

hierarchically reduced from the depth. As $N_{\text {rank }}$ (up to several) is much smaller than $N_{\text {lookup }}$ (dozens) for GnR, the GnR speedup achievable when utilizing rank-level parallelism is fundamentally limited; there is room for further performance improvements by exploiting finer-grained memory-level parallelism.

\subsection{Basic Concept for TRiM}

We present TRiM (Tensor Reduction in Memory), a DRAM-based NDP architecture tailored to the GnR operation. Our proposal is based on the key observation that the datapath constituting any given ranks/bank-groups/banks exhibits a tree-like interconnect topology. TRiM opens up opportunities to conduct the GnR operation hierarchically by employing the NDP-based PE unit per memory node (e.g., a bank, bank-group, or rank). In TRiM, each memory node can perform the GnR operation independently without using the bus closer to the root node (MC). Therefore, TRiM can utilize internal bandwidth equal to the channel bandwidth multiplied by the number of memory nodes $\left(N_{\text {node }}\right)$. By adopting finer-grained memory-level parallelism, $N_{\text {node }}$ increases, and the internal bandwidth increases accordingly.

Embodiments of the DDR4/5-based TRiM architecture include TRiM-R/G/B, corresponding to the depth Rank/bank-Group/Bank to which a $P E$ is allocated. Figure 5 shows the simplified architecture of TRiM-G/B. In TRiM-G, there is a PE per bank-group. The PEs are located inside the DRAM chip so that the bus between the bankgroup and the rank is not used for reduction within bank-groups. Similar to TRiM-G, TRiM-B has a PE for each bank, and the PEs are also located inside the DRAM chip.

To utilize the internal bandwidth provided by TRiM fully, an effective embedding table mapping scheme is needed. As discussed in Section 3.1, different mapping schemes (vP or hP) present different tradeoffs. When TRiM adopts vP, $N_{\text {node }}$ rows must be activated to read a vector. Accordingly, the ACT energy increases. Moreover, the internal bandwidth is wasted when the partitioned vector size is smaller than the minimum DRAM read granularity (64B for DDR4/5). In contrast, when TRiM adopts hP, the C/A bandwidth requirement increases proportionally to $N_{\text {node }}$, and load-imbalance issue arises. One can also imagine a hybrid of $\mathrm{vP}$ and $\mathrm{hP}(\mathrm{vP}-\mathrm{hP})$ when $N_{\text {node }}$ is large enough; for example, $\mathrm{vP}$ is applied between memory nodes in different ranks, whereas $\mathrm{hP}$ is applied between memory nodes in different bank-groups. Unfortunately, such a design point inherits the shortcomings of both $\mathrm{hP}$ and $\mathrm{vP}$ as the ACT energy increases proportionally to $N_{\text {rank }}$ and the C/A bandwidth 


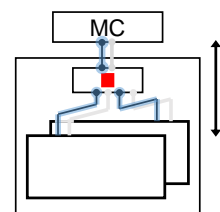

(a)

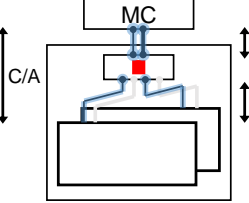

(b)

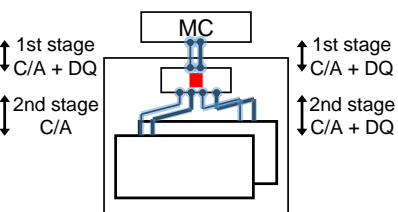

(c)
Figure 6: Various methods for transferring C-instr, the C/A signal to process the GnR operation, to the memory node in the TRiM architecture. (a) Using only C/A pins, adopting a two-stage $\mathrm{C}$-instr transfer scheme and (b) only using $\mathrm{C} / \mathrm{A}$ pins, or (c) C/A and DQ pins together at the second stage.

usage increases proportionally to the number of bank-groups. Moreover, the load imbalance and internal bandwidth waste issues still exist.

Because adopting vP or vP-hP deteriorates the performance and energy efficiency of TRiM, we employ hP as the mapping scheme for the TRiM architecture. Given that RecNMP also employs an hP mapping scheme, we henceforth refer to RecNMP without RankCacahe as TRiM-R. Then, the increase in $N_{\text {node }}$ entails a proportional increase in the $\mathrm{C} / \mathrm{A}$ bandwidth demand. We address the issues of the $\mathrm{C} / \mathrm{A}$ bandwidth shortage and the load-imbalance under TRiMR/G/B using our novel two-stage C-instr transfer and hot-entry replication scheme, the details of which are discussed later in this section.

Figure 3(d) highlights the key benefits of TRiM against prior NDP proposals for RecSys (see Section 4.4 for more details on the TRiM architecture). First, in-memory-node PE for Reduction (IPR) dedicated to each memory node gathers a series of embedding vectors from the corresponding DRAM banks within its local memory node, generating the final (partially) reduced vector in an accelerated manner. Multiples of IPR-reduced vectors are collected by the near-memory-node PE for Reduction (NPR) in the aggregate for the next level of reduction at the parent memory node. The final output reduced from NPR is eventually transferred back to the host $\mathrm{MC}$, and then reduced at the host. Transferring the reduced vectors of a $\mathrm{GnR}$ batch and performing the element-wise $\mathrm{GnR}$ operations of the subsequent $\mathrm{GnR}$ batch can be done in parallel without sharing the datapath of DRAM and hence becoming overlapped.

By conducting the entire in-memory-node reduction operation within the shared tree datapath, our proposed NDP architecture reaps the abundant memory bandwidth (i.e., channel bandwidth $\times N_{\text {node }}$ ) unlocked with in-memory processing, achieving superior performance. Also, TRiM significantly improves the energy efficiency as the data that reaches the NPR of the parent memory node is already partially reduced using our proposed IPR units, reducing the power requirement. Maximally unlocking the potential of in-memory processing requires TRiM to have the ability to supply GnR commands sufficiently to the NDP units. In the following section, we discuss our proposal for amplifying the C/A bandwidth to help realize the maximum degree of memory-level parallelism.

\subsection{How to Provision C/A Bandwidth}

C/A signals for GnR must be sufficiently supplied to all memory nodes in TRiM. We leverage a scheme proposed in RecNMP [29], which compresses ACT, sequential RDs, and PRE into a C-instr. One

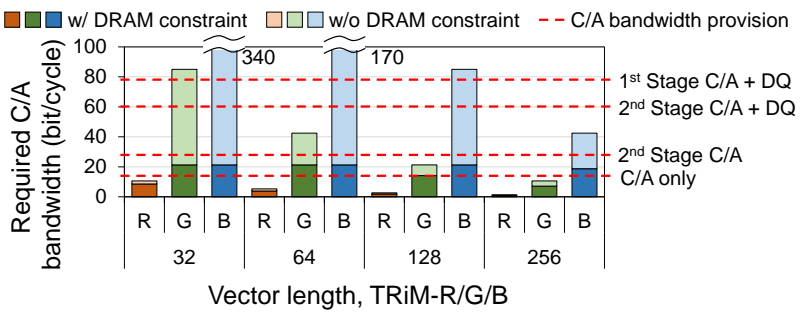

Figure 7: C/A bandwidth requirement to utilize all memory nodes for each TRiM architecture and bandwidth provision for each method of transferring C-instr. In TRiM-G/B, the required bandwidth for $\mathrm{C}$-instr is significantly reduced due to DRAM timing constraints compared to that without these constraints.

C-instr consists of 85 bits and takes charge of one embedding vector lookup. A C-instr is decoded to conventional DRAM commands in the command decoder located in each memory node, which is then transferred to the corresponding bank.

To avoid performance bottlenecks from an insufficient C/A bandwidth, MC must supply $N_{\text {node }} \mathrm{C}$-instrs within the minimum time for a memory node to start processing consecutive $C$-instrs (referred to as $\left.t_{C \text {-instr }}\right)$. Assuming that TRiM fully utilizes the internal bandwidth, $t_{C \text {-instr }}$ is identical to the time to read the vector from a memory node, which is proportional to $v_{l e n}$ and the read cycle. When transferring C-instrs through C/A pins (see Figure 6(a)), the following condition must be met to provide $\mathrm{C}$-instr sufficiently to all memory nodes:

$$
t_{C \text {-instr }} \geq N_{\text {node }} \cdot \frac{C \text {-instr bits }}{C / A \text { bandwidth }}
$$

The right side of Eqn. (1) is the time taken to deliver C-instr to all memory nodes, which is (time to transfer one C-instr) $\times N_{\text {node }}$. In DDR5, the C/A bandwidth is 14 bits/cycle, meaning that $\mathrm{C}$-instr can be sufficiently supplied up to five memory nodes when $v_{l e n}$ is 64. Consequently, only utilizing $\mathrm{C} / \mathrm{A}$ pins cannot provide sufficient bandwidth to TRiM-G/B with multiple ranks. C-instrs can be transferred with a much higher bandwidth by using DQ pins (data pins) in addition to $\mathrm{C} / \mathrm{A}$ pins. If a $\mathrm{C}$-instr passes through the buffer chip and is transferred into a DRAM chip directly, inefficiency arises because only a portion of the DQ pins from the buffer chip are connected to each DRAM chip. Our key approach is to transfer C-instr only to the buffer chip instead (not to the DRAM chip) using the $\mathrm{DQ}$ pins because all DQ pins from the MC are connected to the buffer chip, which helps to amplify the effective C/A bandwidth as follows:

$$
t_{C \text {-instr }} \geq N_{\text {node }} \cdot \frac{C \text {-instr bits }}{\left(D Q_{M C}+C / A\right) \text { bandwidth }}
$$

When the $\mathrm{C}$-instr is transferred from the $\mathrm{MC}$ to the buffer chip using DQ pins and C/A pins together in DDR5, 5.6× more bandwidth (624bit / 8 cycle) can be utilized than when transferring a C-instr using $\mathrm{C} / \mathrm{A}$ pins only.

We propose a two-stage $C$-instr transfer scheme that delivers a Cinstr through a path from the MC to the buffer chip, using another path from the buffer chip to the DRAM chip. In the first stage, a $\mathrm{C}$-instr is transferred using $\mathrm{C} / \mathrm{A}$ pins and DQ pins together. In the 
second stage, we consider two design points (see Figures 6 (b) and (c)), to use only C/A pins (Eqn. (3)), or to use C/A pins and DQ pins together (Eqn. (4)). These two stages can be pipelined as they do not share a datapath. That is, all buffer chips, which have dedicated data/control paths connecting the ranks, can independently transfer $\mathrm{C}$-instrs to the DRAM chip. Thus the aggregate $\mathrm{C} / \mathrm{A}$ bandwidth of the second stage grows proportionally to $N_{\text {rank }}$.

$$
\begin{gathered}
t_{C \text {-instr }} \geq \frac{N_{\text {node }}}{N_{\text {rank }}} \cdot \frac{C \text {-instr bits }}{C / A \text { bandwidth }} \\
t_{C \text {-instr }} \geq \frac{N_{\text {node }}}{N_{\text {rank }}} \cdot \frac{C \text {-instr bits }}{\left(D Q_{\text {chip }}+C / A\right) \text { bandwidth }}
\end{gathered}
$$

When sending a $\mathrm{C}$-instr, using both $\mathrm{C} / \mathrm{A}$ and $\mathrm{DQ}$ pins can utilize more bandwidth than using only $\mathrm{C} / \mathrm{A}$ pins. However, if $\mathrm{C} / \mathrm{A}$ and DQ pins are used together, there may be a conflict between the transfer of a partially reduced vector from the IPR to the NPR and the transfer of $\mathrm{C}$-instr. This can also cause an additional delay due to the bus turnaround of the datapath. Thus, if Eqn. (3) is satisfied, it is better to use only $\mathrm{C} / \mathrm{A}$ pins in the second stage.

When two DDR5 ranks are populated per memory channel, we measure the C/A bandwidth requirement in TRiM-R/G/B by changing $v_{\text {len }}$ from 32 to 256 and the bandwidth provision according to the C-instr supply method used (see Figure 7). The light bar graph in Figure 7 is the $\mathrm{C} / \mathrm{A}$ bandwidth requirement without any constraint when the DRAM access size and read cycle are set to $(64 \mathrm{~B}, 8)$, and the dark bar graph is the $\mathrm{C} / \mathrm{A}$ bandwidth requirement when considering the various timing constraints (e.g., tRRD and tFAW). The red dotted lines represent the bandwidth provision according to the $\mathrm{C}$-instr supply method: using 1 ) only $\mathrm{C} / \mathrm{A}$ pins as in a conventional DRAM (C/A only), 2) C/A and DQ pins to a buffer chip (1st stage $\mathrm{C} / \mathrm{A}+\mathrm{DQ}$ ) but only $\mathrm{C} / \mathrm{A}$ pins from the buffer chip (2nd stage $\mathrm{C} / \mathrm{A}$ ), and 3 ) $\mathrm{C} / \mathrm{A}$ and $\mathrm{DQ}$ pins from the buffer chip (2nd stage $\mathrm{C} / \mathrm{A}+\mathrm{DQ})$.

The larger the $v_{l e n}$, the longer it takes to process one C-instr; thus, the $\mathrm{C} / \mathrm{A}$ bandwidth requirement decreases. When the DRAM timing constraints are not considered, the bandwidth requirement inversely decreases proportionally to $v_{l e n}$. In TRiM-G/B, the required C/A bandwidth for a $\mathrm{C}$-instr is significantly reduced by the DRAM constraints especially those limiting frequent activations within the rank, which consequently limit the multiple memory nodes in the same rank from operating in parallel.

The two-stage C-instr transfer scheme is compelling for increasing the effective bandwidth for $\mathrm{C}$-instr (more than $2 \times$ compared to when using $\mathrm{C} / \mathrm{A}$ pins only). We choose to use only $\mathrm{C} / \mathrm{A}$ pins in the second stage because the $\mathrm{C} / \mathrm{A}$ bandwidth provision of this approach is sufficient to fully supply C-instrs for TRiM-R/G/B with $v_{l e n}$ from 32 to 256.

\subsection{Exploring NDP Unit Placement}

The optimal TRiM design point varies depending on the characteristics of the GnR workload. Allocating the PE for a certain memory depth allows GnR to exploit the internal bandwidth up to the channel bandwidth multiplied by $N_{\text {node }}$ in a memory channel. However, if the load is not sufficiently distributed to the PE or the time to read an embedding vector is relatively short, TRiM could suffer from low utilization of the internal DRAM data transfer bandwidth.

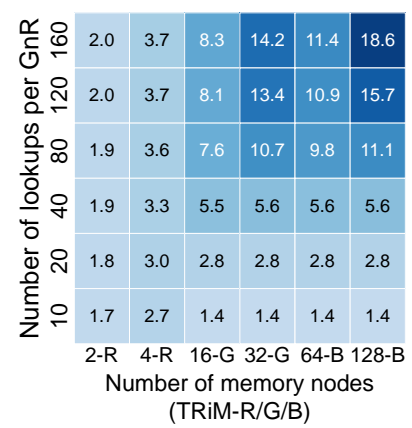

(a)

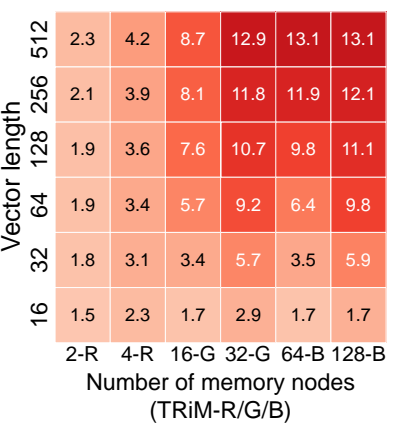

(b)
Figure 8: Heatmaps showing the speedup of TRiM-R/G/B over Base (a) according to $N_{\text {lookup }}$ when $v_{l e n}$ is 128 and (b) according to $\boldsymbol{v}_{\text {len }}$ when $\boldsymbol{N}_{\text {lookup }}$ is 80 . The number of memory nodes of TRiM-R/G/B is $2 / 16 / 64$ in 1 DIMM $\times 2$ ranks and $4 / 32 / 128$ in 2 DIMM $\times 2$ ranks.

In Figure 8, we measure the throughput of GnR compared to Base according to $N_{\text {node }}$ while varying $N_{\text {lookup }}$ and $v_{\text {len }}$, assuming 2/4-rank DDR5-4800. In the TRiM architecture, the performance of GnR improves as $N_{\text {node }}$ increases because the available aggregate bandwidth increases proportionally to $N_{\text {node }}$ as each memory node can utilize a dedicated datapath. Therefore, the rightmost end of the heatmaps with the largest number of memory nodes performs best.

However, the speedup saturates when adopting excessive parallelism. When multiple banks share a datapath, one bank can occupy the datapath by reading data while the other banks are preparing data, meaning that the data preparation time can be hidden and the internal bandwidth can be highly utilized. Using finer-grained parallelism reduces the number of banks in one memory node and thus reduces the number of banks sharing the datapath. In this case, the data preparation time might not be completely hidden and the utilization rate deteriorates. This demerit is pronounced when $v_{\text {len }}$ is low, where the ratio of the data preparation time is relatively high, as shown in the bottom right of Figure 8(b). Also, limiting the frequency of activation in DRAM chips saturates the performance improvement as $N_{\text {node }}$ increases.

To utilize the aggregate bandwidth obtained by increasing $N_{\text {node }}$ fully, $N_{\text {lookup }}$ should be sufficiently large. When this is the case, the time to perform a reduction in the memory node overlaps with the time for transferring the partially reduced vector to the PE at the parent memory node. Then, the speedup is eventually bounded by the ratio of the internal aggregate bandwidth in TRiM over the channel bandwidth. In contrast, if $N_{\text {lookup }}$ is too small, the time to undertake reduction in the memory node is completely overlapped by the time for the transfer of the partially reduced vector to the $\mathrm{PE}$ at the parent node, limiting the speedup (see the lower right part in Figure 8(a)).

Considering the results in Figure 8, TRiM-G is most efficient, in common RecSys models with $v_{l e n}$ of 20-80 and $N_{\text {node }}$ of 32256. Although TRiM-B shows better performance at some points, it incurs over $4 \times$ more area overhead than TRiM-G as the NDP unit is employed per bank, not per bank-group. As this area overhead can also lead to greater energy overhead to keep the DRAM read/write 


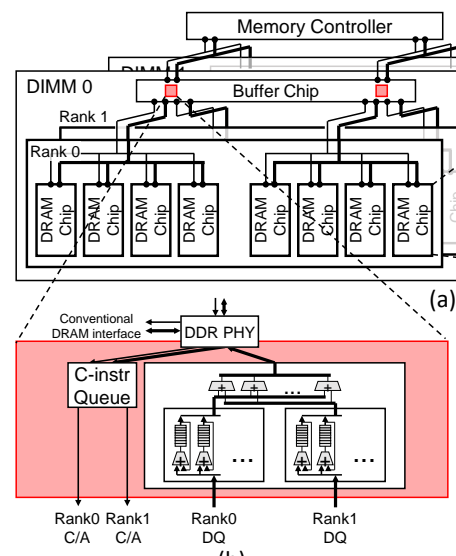

(b)
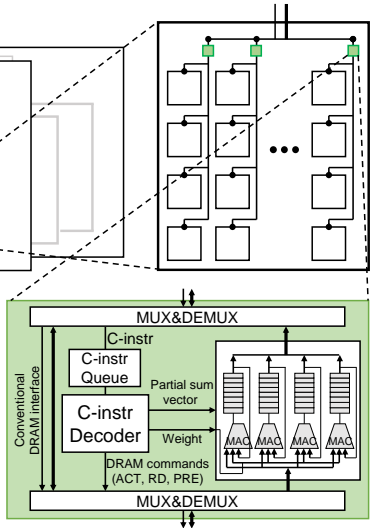

(c)
Figure 9: (a) Overall architecture of TRiM-G, (b) NPR, and (c) IPR assuming an $\times 8$ data I/O bit-width. Four 32 -bit MACs are placed in the IPR.

latency unchanged $[49,59]$, we consider TRiM-G as a better option compared to TRiM-B.

We set TRiM-G with 16 memory nodes as the default configuration. When $N_{\text {lookup }}$ is 80 , TRiM-G with 32 memory nodes (2 DIMMs $\times 2$ ranks) performs better than TRiM-G with 16 memory nodes (1 DIMM $\times 2$ ranks). However, if the lookups are evenly distributed across the memory nodes, we observe that there is no significant difference in performance between these two TRiM-G configurations. The load-imbalance issue is mitigated by applying hot-entry replication, which will be described later. Then, in DDR5, an embedding table is stored only in 1 DIMM $\times 2$ ranks $\times 8$ bank-groups, allowing multiple embedding tables to be looked up concurrently where performance improvements can be multiplied by the number of DIMMs.

Designing TRiM "in-memory" (rather than "near-memory" at the buffer chip) allows the utilization of the abundant internal DRAM bandwidth while consuming much less energy thanks to the shorter datapath. The trade-off lies in the higher area overhead of the "inmemory" approaches; DRAM processes with fewer metal layers require more area for designing logic elements, and the power and timing constraints of a DRAM chip hinder the maximal use of its internal bandwidth.

\subsection{TRiM-G Organization and Operations}

Based on our design space exploration, this section presents our TRiM-G architecture (see Figure 9), where an NDP unit tailored for $\mathrm{GnR}$ is employed per bank-group as well as per rank. IPR for TRiM-G is located between the bank-group I/O multiplexer (MUX) and the global I/O MUX. NPR for TRiM-G is placed on the buffer chip. IPR consists of 32-bit floating-point multiply-add (add for NPR) units (MACs) for vector reduction and includes registers to store the partial reduction of the vectors. Each buffer chip has a queue for the temporary storage of the $\mathrm{C}$-instrs transferred from MC. The C-instr decoder is located in the IPR to decode a C-instr to DRAM commands (ACT, RD, and PRE) and to send internal DRAM commands to each bank considering bank interleaving.
One 85-bit C-instr consists of target-address (34-bit), weight (32bit), $n R D$ (5-bit), batch-tag (4-bit), opcode (3-bit), skewed-cycle (6bit), and vector-transfer (1-bit). The target-address is the starting address of a vector. The weight contains 32-bit floating-point data for supporting the weighted-sum operation. The $n R D$ is the number of DRAM read commands per vector, which also indicates the size of the vector to be processed per C-instr. The batch-tag identifies the $\mathrm{C}$-instr belonging to the same $\mathrm{GnR}$ operation in a GnR batch. The opcode determines the type of element-wise reduction operation (e.g., sum or weighted sum). The skewed-cycle indicates when the current $\mathrm{C}$-instr starts operation at the memory node after arrival, and the vector-transfer instructs each memory node to transfer a partially reduced vector to the parent memory node. The vectortransfer bit is set to 1 for the last $\mathrm{C}$-instr in the batch.

TRiM-G operates as follows. At the first stage of the two-stage C-instr transfer, up to $7 \mathrm{C}$-instrs enter the $\mathrm{C}$-instr queue of the NPR in the buffer chip for every eight cycles exploiting C/A and DQ paths. In the C-instr queue of the NPR, a C-instr is transferred to the $\mathrm{C}$-instr queue of the IPR in the target bank-group through the C/A path in order (the second stage). For each C-instr in the queue, the decoder in the IPR starts sending DRAM commands (i.e., ACT, PRE, and RDs) into the internal DRAM after the skewed-cycle. Whenever a RD is sent, the MAC units in the IPR accumulate data after a delay of tCL (access time). If the C-instr with the vector-transfer bit set enters the NPR queue, the NPR alternately sends commands to each IPR to transfer a partially reduced vector to the NPR by occupying the depth- 2 data bus. After the first of these commands is sent, the adders in the NPR accumulate the partial sum of each IPR for every tCCD. The command for transferring a partially reduced vector is defined using reserved-for-use (RFU) commands. When accumulation for each rank is completed, other adders in the NPR combine the partial sums of the ranks. Finally, the MC reads the partial sums of the DIMMs.

\subsection{Host-side Architecture for TRiM}

Hot-entry Replication for Balancing Loads: Figure 10 shows the distribution of the maximum loads (the number of lookups) across the memory nodes per GnR, normalized to a perfectly balanced load. The performance of TRiM is bound to the memory node that has the largest number of lookups. As $N_{\text {node }}$ increases, the number of lookups for a single memory node decreases, but the load imbalance becomes more severe. RecNMP alleviates this problem by batching $\mathrm{GnR}$ operations, processing multiple GnR operations at one time. Batching incurs an area overhead that is proportional to $N_{G n R}$ because dedicated registers are required to hold a partial reduction of vectors. Applying a batch of $8 \mathrm{GnR}$ operations to TRiM-G causes an additional $2.5 \%$ of DRAM chip overhead.

We tackle the load-imbalance challenges with our novel hotentry replication scheme, which copies frequently accessed (hot) entries to each memory node; this scheme is motivated by the replication method often used in datacenters [47]. We observe that a few entries occupy a large portion of the lookup requests (more details about the dataset are given in Section 5). Load balancing is done by distributing the lookup request, which corresponds to the C-instr. Only hot requests, ones that head toward hot entries, are redirected to the replicated entries at the nodes with lower loads. 


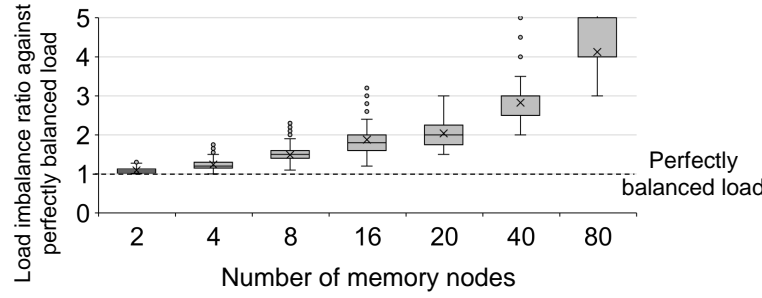

Figure 10: Distribution of the load imbalance ratio, the largest number of lookups among memory nodes in each GnR, normalized to the number of lookups of a memory node assuming that loads are evenly distributed to all memory nodes. $\boldsymbol{N}_{\text {lookup }}$ is $\mathbf{8 0}$.

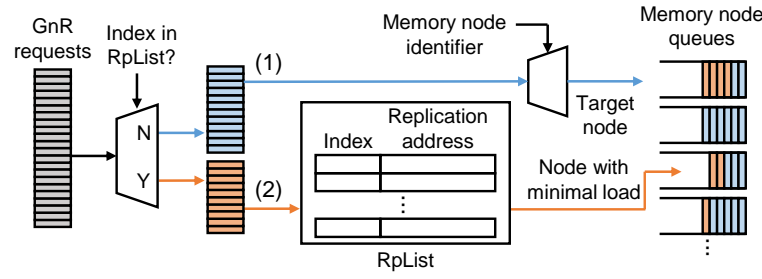

Figure 11: Execution flow of lookup request distribution through hot-entry replication.

Hot entries are statically determined by profiling embedding table access traces, after which they are replicated and stored at the same address (bank, row, column for TRiM-G) in each memory node.

Figure 11 illustrates the execution flow for the distribution of lookup requests. There is a list of replicated entries (RpList), where the replicated entries exist at the same relative locations across all memory nodes. All lookup requests in a GnR batch are classified based on whether the target index of the request is on the RpList. Lookup requests other than hot requests are put in the request queue dedicated to each memory node. Then, hot requests are distributed to the queue of the memory node with the minimal load.

There is a trade-off between the number of hot entries $\left(N_{h o t}\right)$ and the speedup because the load balance improves as $N_{\text {hot }}$ increases, but the capacity overhead rises (proportional to $N_{\text {node }}$ ). Because both hot-entry replication and batching mitigate the load-imbalance issue, $N_{h o t}$ and $N_{G n R}$ should be configured considering the area and memory capacity overheads.

Storing hot-entries in the host cache can improve the performance of $\mathrm{GnR}$ operations, but hot-entry replication is preferred for the following reasons. First, the degree of performance improvement by the host cache is limited because only a small portion of entries can be stored in the cache. The embedding table corresponding to a size of hundreds of GBs can be stored in the main memory, whereas the size of the host cache is few tens of MBs; only about a hundredth of a percent of the embedding table can be stored in the cache. Second, even if the performance is improved by the method described above, it could degrade the performance of other operations. As found in earlier work [29], if hot entries are stored in the cache, data such as the weights of the FC layer required for the RecSys model can be evicted. Consequently, this not only reduces

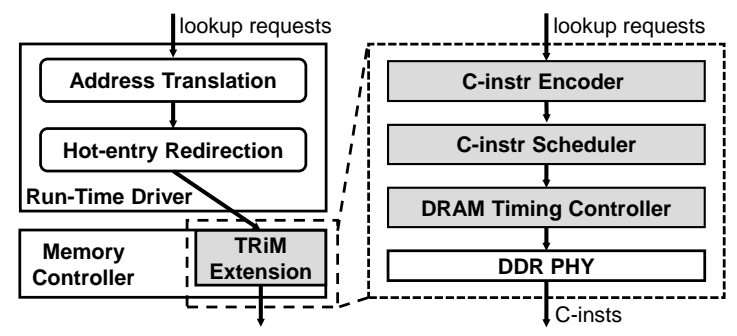

Figure 12: An overall execution flow of the lookup requests on the host-side.

the performance of other operations, but also increases the latency. With TRiM, this effect is even greater because FC takes up most of the time.

RecNMP uses the cache in the buffer chips to exploit the temporal locality of hot entries. However, using the cache on the DRAM side breaks the conventional interface by which the DRAM access latency is deterministic, making it unsuitable for TRiM. Also, transferring signals for cache hits from DRAM to MC is expensive as polling is required and an additional scheduler should exist per memory node.

Programming, Memory Model, and Data Placement: TRiM leverages the programming and memory models in previous NDP architectures $[6,14,29]$. The host runs a RecSys application and offloads a portion of the GnR operation to TRiM, similar to CUDA [44]. By ensuring that the contiguous virtual address range for an embedding table is mapped to a contiguous physical address range, the physical address of each embedding vector index can be identified from the starting address of the embedding table stored in a TRiM-specific driver. When placing the embedding table in DRAM, the TRiM-specific driver evenly distributes the embedding table to the memory nodes exploiting DRAM address mapping. The host and TRiM have their own independent memory region within one physical memory space to avoid memory consistency issues. Following [6,14], we address cache coherence issues by marking the memory region for TRiM as uncacheable and shuffle data in an embedding table entry to place a consecutive 32-bit floating-point value into a DRAM chip for the IPR.

Execution Flow on the Host side: Figure 12 shows how lookup requests are processed on the host side prior to being transferred to TRiM. As $N_{\text {hot }}$ is configurable, the RpList can be enlarged according to the embedding table size and the replication rate of hot-entry replication $\left(p_{\text {hot }}\right)$, representing a burden to put the RpList on the MC. Accordingly, we assign a run-time driver tailored for TRiM to distribute hot requests. It receives lookup requests and undertakes the distribution of the hot requests. The lookup requests are transferred to the MC with the TRiM extension, and the C-instr encoder encodes the requests to $\mathrm{C}$-instrs. Then, the $\mathrm{C}$-instr scheduler reorders the $\mathrm{C}$-instrs for each $\mathrm{GnR}$ batch considering that multiple memory nodes operate simultaneously. After the completion of a scheduling task for a $\mathrm{GnR}$ batch, the DRAM timing controller sets the skewed cycle in the $\mathrm{C}$-instrs. The $\mathrm{C}$-instrs are then transferred to the DRAM, obeying the DRAM timing constraints. 
Table 1: Timing/energy parameters of $16 \mathrm{~Gb}$ DDR5-4800 $\times 8$ DRAM chips and NDP units.

\begin{tabular}{lc}
\hline Parameters & Values \\
\hline Clock frequency (1/tCK) & $2,400 \mathrm{MHz}$ \\
Cycle time (tRC) & $48.64 \mathrm{~ns}$ \\
ACT to RD, Access, PRE time (tRCD, tCL, tRP) & $16.64 \mathrm{~ns}$ \\
Read to read between different bank-groups (tCCD_S) & $8 \mathrm{tCK}$ \\
Read to read to the same bank-group (tCCD_L) & $12 \mathrm{tCK}$ \\
Four activate window (tFAW) & $13.31 \mathrm{~ns}$ \\
ACT energy & $2.02 \mathrm{~nJ}$ \\
On-chip read/write energy & $4.25 \mathrm{pJ} / \mathrm{b}$ \\
Read energy to bank-group (BG) I/O MUX & $2.45 \mathrm{pJ} / \mathrm{b}$ \\
Off-chip I/O energy & $4.06 \mathrm{pJ} / \mathrm{b}$ \\
MAC unit energy in IPR & $3.23 \mathrm{pJ} / \mathrm{Op}$ \\
Adder energy in NPR & $0.90 \mathrm{pJ} / \mathrm{Op}$ \\
\hline
\end{tabular}

\subsection{Schemes for Improving Reliability}

In TRiM-G/B, the GnR operation is performed inside the DRAM chip and hence the conventional rank-level error correction code (ECC) cannot be applied for error detection and correction, necessitating a different way to ensure reliability when reading data for GnR inside a DRAM chip. Modern DRAM chips have started to adopt on-die ECC, which operates inside the DRAM chip (die) to improve data integrity further $[7,48,51,52,58]$. In particular, DDR5 uses single-bit error correction (SEC) codes for the on-die ECC [26]. However, the reliability of DRAM only with conventional on-die ECC (SEC) is lower than that with rank-level ECC (typically supporting SECDED, SEC with double-bit error detection (DED)) and on-die ECC together.

To improve the reliability of data being read during GnR to a level equivalent to DED, we propose to repurpose the on-die SEC code to detect double-bit errors. This is possible because the embedding tables are read-only while performing GnR and the hamming code used for SEC has a minimal hamming distance of 3, which can be used to detect double-bit errors if correction is not needed [53]. During $\mathrm{GnR}$, the parity bits are calculated for the embedding table entry being read, similar to a normal DRAM write, and those calculated are compared to the stored parity bits. If a mismatch is identified, an error is reported, and the table entry should be reloaded from storage. The overhead of supporting DED for TRiM is minimal as most of the ECC logic is reused; only a simple comparator is added to detect a mismatch between the parity bits.

\section{EXPERIMENTAL SETUP}

Simulation framework: We modified Ramulator [34] to evaluate the performance and energy consumption of TRiM compared to those of the baseline system (Base) and two state-of-the-art NDP architectures, RecNMP and TensorDIMM. The C-instr generator, decoder, and hot-entry replication module are implemented inside our Ramulator-based simulation framework. We set Base and the NDP architectures to use commodity DRAM modules, DDR5-4800, 1 DIMM with 2 ranks per memory channel (see Table 1). Base was simulated in the CPU trace-driven mode with $32 \mathrm{MB}$ of lastlevel cache, which is large enough to saturate the performance improvement due to the temporal locality in our synthetic traces.

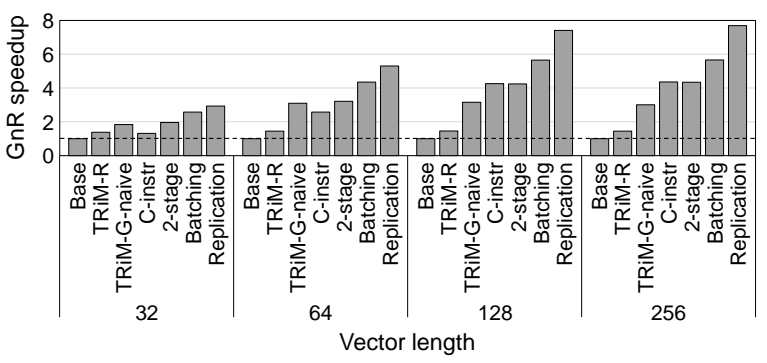

Figure 13: The GnR speedup of the TRiM architectures when applying the TRiM-R, TRiM-G-naive (rank-/bank-grouplevel parallelism), $\mathrm{C}$-instr (instruction compression), 2-stage (2-stage C-instr transfer), Batching (GnR operation batching), and (hot-entry) Replication schemes.

Benchmarks: We utilize representative RecSys models published in prior work [20]. The vector reduction operation of GnR utilizes an element-wise sum operator (SLS) and $N_{\text {lookup }}$ is set to 80 with 32-bit floating-point elements. Following [62], we vary $v_{\text {len }}$ from 32 to 256 in our experiments. Because the real trace used in prior works $[20,29]$ is not publicly available, we generate a synthetic embedding table access trace with the algorithm discussed in [46] using the publicly available Criteo dataset $[9,54]$. Our synthetic trace shows temporal locality similar to the traces presented in $[13,29]$. We set $N_{G n R}$ to 4 and the default phot rate to $0.05 \%$.

Power and area: To analyze the power and area for the NDP architectures including TRiM, we estimate the power consumption of DDR5 DRAM with the DDR4 datasheets from industry [45, 55] and the off-chip I/O with CACTI [27]. We calculated the power of the DRAM read for the IPR (read-only up to bank-group I/O) by referring to FGDRAM [50] and scaled it properly considering the DRAM page size, the number of banks, and the datapath length. We first synthesized both the IPR and NPR units using the Synopsys Design Compiler with 40nm CMOS technology at frequencies of $200 \mathrm{MHz}$ (for IPR) and $300 \mathrm{MHz}$ (for NPR) respectively, which is demonstrated to operate in the other recent NDP architecture implemented in the real DRAM devices [38]. Then, we scaled the result of the IPR to a $20 \mathrm{~nm}$ DRAM process assuming that a DRAM process is $10 \times$ less dense compared to an ASIC process of an equivalent feature size considering fewer metal layers and slower transistors, referring to $[11,57]$.

\section{EVALUATION}

This section initially evaluates the effect of TRiM's various design optimizations by incrementally applying them on top of Base. We then quantify the performance and energy-efficiency benefits of TRiM-G over TensorDIMM and RecNMP. We also analyze the speedup of TRiM over various $p_{h o t}$ values and evaluate the effects of replication and batching together.

\subsection{Performance and Energy Efficiency}

Figure 13 shows the speedup in six scenarios of TRiM when gradually applying the modified design of the C/A signals and optimizations at various values of $v_{l e n}$. The first two scenarios are 


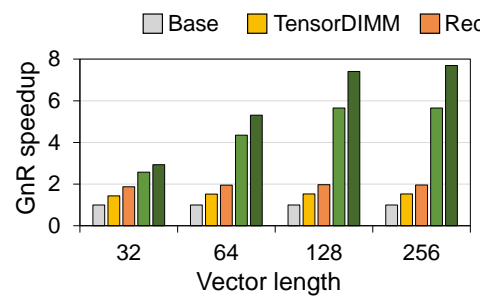

(a)

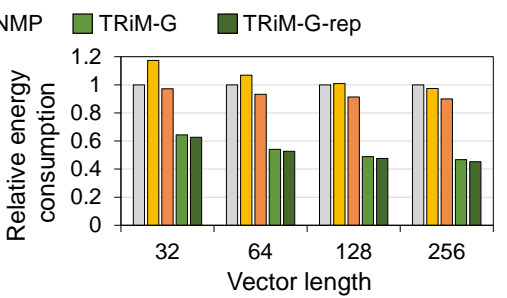

(b)

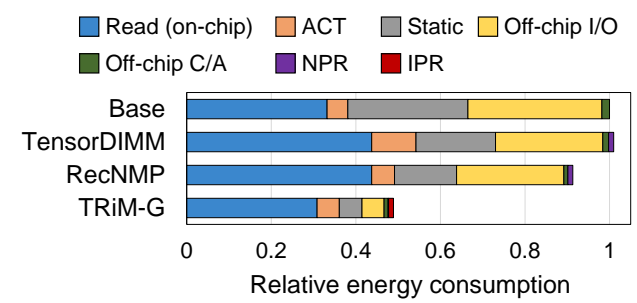

(c)

Figure 14: (a) GnR speedup and (b) relative DRAM energy consumption of TensorDIMM, RecNMP, TRiM-G, and TRiM-G with hot-entry replication (TRiM-G-rep), and (c) energy consumption breakdown when $v_{l e n}$ is 128.

TRiM-R and TRiM-G-naive, each corresponding to the TRiM architecture with rank or bank-group level parallelism without any change in the $\mathrm{C} / \mathrm{A}$ interface. The next two scenarios are $\mathrm{C}$-instr and 2-stage, each applying the instruction compression scheme (proposed in [29]) and the two-stage C-instr transfer scheme to increase the effective C/A bandwidth. The last two scenarios are Batching and Replication, batching GnR operations (proposed in [29]) and a hot-entry replication scheme to alleviate the loadimbalance issue. In later experiments, 2-stage and Replication correspond to TRiM-G and TRiM-G-rep, respectively.

In general, the effectiveness of our proposal is clearly demonstrated by the gradually increasing performance as additional optimizations are incrementally applied, but with varying degrees of effectiveness under different $v_{\text {len }}$ values. TRiM-R improves the performance by up to $1.46 \times$ over Base. TRiM-R can utilize up to twice $\left(N_{\text {rank }}\right)$ as much internal bandwidth relative to that by Base. However, TRiM-R cannot benefit from the cache in the host; thus, its speedup is less than the increase in the internal bandwidth. TRiM-G-naive also uses $8 \times$ more memory nodes and $8 \times$ more internal bandwidth than TRiM-R, but its performance is only slightly higher than that by TRiM-R. This occurs because TRiM-G-naive is more strictly affected by the C/A bandwidth limitation and DRAM constraints than TRiM-R.

C-instr achieves up to $45 \%$ higher performance than TRiM-Gnaive with various values of $v_{l e n}$ because more DRAM commands can actually be transferred by exploiting the instruction compression scheme. However, if $v_{l e n}$ is 32 or 64 , the performance of $\mathrm{C}$-instr is degraded because the number of cycles sending one lookup request by ACT-RDs-PRE is smaller than that by C-instr. 2 -stage improves the performance by $50 \%$ and $24 \%$ when $v_{l e n}$ is 32 and 64, respectively, as it can amplify the bandwidth for the $\mathrm{C}$-instr transfer, which is limited when $v_{l e n}$ is low. Compared to TRiM-G-naive, the schemes for increasing the effective C/A bandwidth achieve a speedup of $1.2 \times$ on average. There is an additional speedup of $67 \%$ on average via batching GnR operations (Batching) and hot-entry replication (Replication), mitigating the load-imbalance issue. In conclusion, TRiM-G applying all of the optimizations achieves speedups of up to $7.7 \times$ over Base and up to $5.3 \times$ over TRiM-R.

TRiM-G improves the performance over TensorDIMM and RecNMP owing to the increased internal bandwidth realized by exploiting finer bank-group-level parallelism. Figure 14 shows the speedup and relative DRAM energy consumption for Base, TensorDIMM, RecNMP, and TRiM-G when varying $v_{\text {len }}$. We linearly scale the speedup and static energy from RankCache in a previous study [29] to the results

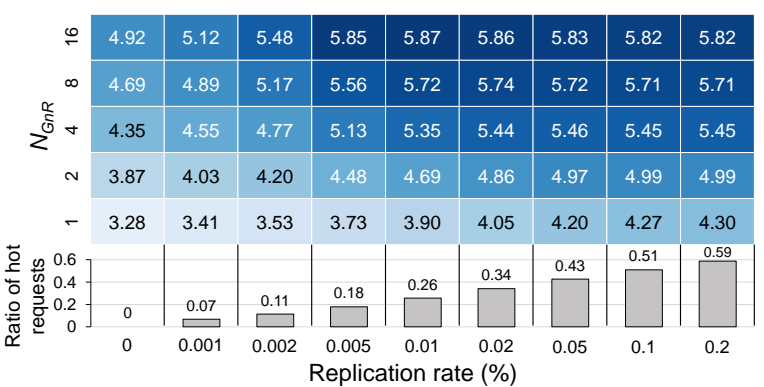

Figure 15: Speedup of TRiM-G over Base with various $p_{h o t}$ and $N_{G n R}$ values (heatmap), the ratio of hot requests over all requests (bar graph), averaged over speedups while varying $v_{l e n}$ from 32 to 256 .

here. TRiM-G achieves a speedup of up to $5.7 \times$ over Base, and up to $3.7 \times$ and $2.9 \times$ over TensorDIMM and RecNMP, respectively (see Figure 14(a)). Despite the fact that $N_{\text {node }}$ of TRiM-G is $8 \times$ larger than that of TensorDIMM and RecNMP, the speedup of TRiM-G is lower than $8 \times$ because the frequency inside a bank-group bus is lower than that outside a bank-group, which reduces the peak bandwidth by $33 \%$, and the load imbalance becomes worse due to the increase in $N_{\text {node }}$. When $v_{\text {len }}$ is 32 and 64, the speedup is relatively low due to the limitation of the ACT frequency, while TRiM-G demands more frequent ACTs for higher bandwidth utilization. If $v_{\text {len }}$ exceeds 128 , the speedup is nearly saturated by the internal bandwidth.

As shown in Figure 14(b), TRiM-G consumes up to 55\%, 54\%, and $50 \%$ lower DRAM energy than Base, TensorDIMM, and RecNMP, respectively. Figure 14(c) shows the energy consumption breakdown when $v_{\text {len }}$ is 128 . TRiM-G dissipates $7 \%$ less on-chip read energy than Base with the host-side cache. Compared to RecNMP, TRiM-G consumes $30 \%$ less on-chip read energy and $79 \%$ less off-chip I/O energy due to the decreased data transfers from the IPR to the NPR, and $63 \%$ less static energy due to the reduced execution time. The energy consumption by the NPR and IPR for TRiM-G is negligible, accounting for $0.24 \%$ and $2.47 \%$, respectively.

Hot-entry replication can improve the performance by up to $36 \%$ by alleviating the load imbalance. TRiM-G with hot-entry replication achieves a speedup of up to $7.7 \times$ over Base, and up to $5.0 \times$ and $3.9 \times$ correspondingly over TensorDIMM and RecNMP (see Figure 14(a)). The impact of hot-entry replication on the energy efficiency is negligible because this scheme does not change the total number of lookups in GnR (see Figure 14(b)). 


\subsection{Sensitivity Study of Hot-entry Replication}

Increasing $p_{\text {hot }}$ can improve the performance, as the load balancing improves. In Figure 15, the heatmap shows the speedup of TRiM-G over Base according to $N_{G n R}$ and $p_{\text {hot }}$, averaged over the speedups while $v_{\text {len }}$ ranges from 32 to 256 . The bar graph shows the ratio of hot requests to all requests according to $p_{\text {hot }}$. As more requests are distributed to memory nodes with lower loads as $p_{h o t}$ increases, the load imbalance is alleviated. With $p_{\text {hot }}$ set to $0.05 \%$ and with a hot request rate of $42 \%$, the speedup is nearly saturated, being $0.2 \%$ lower than that for a perfectly balanced workload and $25 \%$ higher than TRiM-G without hot-entry replication when $N_{G n R}$ is set to 4 .

Because increasing $N_{G n R}$ (batching) and phot (hot-entry replication) causes area and memory capacity overhead, both should be carefully selected in consideration of the diminishing return of speedup. If $N_{G n R}$ exceeds 8 without replication, the speedup is saturated to around 5.0. With a small $p_{\text {hot }}$ of hot-entry replication, the speedup surpasses 5.0 when $N_{G n R}$ is 4 . Thus, we set $N_{G n R}$ to 4 considering that larger values return a low-performance gain with a large register file requirement, which degrades the DRAM access latency further. Also, we set $p_{\text {hot }}$ to $0.05 \%$ such that it saturates the speedup and incurs only $0.8 \%$ of the memory capacity overhead.

\subsection{Design Overhead}

The total area overhead of IPR is $2.03 \mathrm{~mm}^{2}$ per $16 \mathrm{~Gb}$ DDR5 DRAM die [33], which corresponds to $2.66 \%$, assuming that each chip has a $\times 8$ data I/O bit-width and $\left(v_{l e n}, N_{G n R}\right)$ is $(256,4)$. Each IPR, one per bank-group, includes four MACs and two $1 \mathrm{~KB}$ register files considering double buffering. Because adding an IPR incurs a small amount of overhead, increasing the driver strength of the interbank datapath by a small degree keeps the DRAM access latency unchanged with a minimal increase in the read/write energy [59]. The area of the NPR is $0.361 \mathrm{~mm}^{2}$, similar to the area of RecNMP without RankCache.

\section{DISCUSSION}

Applying TRiM to Matrix-Vector Multiplication: TRiM can also be applied to the general matrix-vector multiplication (GEMV) operations commonly used in the FC layers of neural networks [38]. The reusability of weight matrices in GEMV is relatively low such that the performance can be bottlenecked by the memory bandwidth, which is appropriate to accelerate by NDP architectures. TRiM can store the weight matrices in DRAM cells and receive the input vectors from the host, temporarily storing them in the buffer. The register files for storing the partial sums of vectors in IPR can be used as the buffer for temporarily storing the input/output vectors for the GEMV operation in each memory node. With proper support from the software stack, TRiM can accelerate the memorybound GEMV by fully exploiting the internal aggregate bandwidth of DRAM devices.

\section{RELATED WORK}

Accelerating RecSys: The computer systems community has recently seen growing interest in accelerating RecSys [18-20, 22, 23, 28-30, 35, 61]. FAFNIR [5] exploits a tree structure to perform all reductions in rank-level parallel NDP units, reducing the off-chip data movement and the number of connections between the cores and the NDP units. However, FAFNIR requires a separate chip outside of the DRAM module and is not compatible with the memory controller on the conventional processor die. Tensor Casting [36] proposed a rank-level parallelism NDP architecture for training RecSys models. In addition to the method of accelerating embedding layers, [56] exploited complementary partitions that reduced the number of embedding vectors while preserving the uniqueness of the embedding, and [63] proposed a low-precision embedding table with a high-precision cache.

Near-Data Processing: A large body of prior work has sought to place the processing logic closer to the memory. Similar to TRiM, recent works proposed DRAM or DIMM module-based near-data processing architectures [3, 4, 14, 21, 40, 41, 43, 57]. Chameleon [6] proposed an NDP architecture based on Load-Reduced DIMM. Adding logic to stacked memory solutions was also explored in prior works [12, 15, 17, 32, 39, 42]. TETRIS [16] adds a simple data accumulator near the DRAM banks to accelerate the CNNs, but the proposed architecture cannot fully utilize the internal DRAM bandwidth. [37] presents an HBM processing-in-memory chip that exploits bank-level parallelism. However, this architecture is inefficient when used to perform reduction operations because it neither organizes PEs hierarchically nor allows PEs to access non-local memory. To the best of our knowledge, TRiM, which extended [31], is the first proposal to accelerate hierarchical reduction while exploiting the tree-like interconnect topology of DIMM-based main memory.

\section{CONCLUSION}

We have proposed TRiM, a near-data processing (NDP) architecture for accelerating tensor gather-and-reduction $(\mathrm{GnR})$ operations in recommendation systems. First, we identified the challenges of state-of-the-art NDP architectures for accelerating GnR and the potential for further energy-efficiency improvements by unlocking the inherent bandwidth amplification opportunities within the DRAM chip's tree-topology-based datapath by populating processing elements for a reduction at the datapath. We proposed a two-stage instruction transfer scheme to amplify the control bandwidth by splitting the data path into two stages and pipelining them. The hot-entry replication scheme alleviates the load imbalance problem. We also improve the data reliability of TRiM by repurposing the existing on-die ECC to only detect and not correct errors during GnR as it accesses the embedding tables in a read-only manner. TRiM improves the performance of $\mathrm{GnR}$ by up to $7.7 \times$ and $3.9 \times$ compared to the DDR5-based baseline system and the state-of-the-art NDP architecture, respectively.

\section{ACKNOWLEDGMENTS}

This research was supported by the Engineering Research Center Program through the National Research Foundation of Korea (NRF) funded by the Korean Government MSIT (NRF-2018R1A5A1059921) and the R\&D program of MOTIE/KEIT (10077609, Developing Processor-Memory-Storage Integrated Architecture for Low Power, High Performance Big Data Servers). Jung Ho Ahn is the corresponding author. 


\section{REFERENCES}

[1] 2017. Caffe2. https://caffe2.ai

[2] Jung Ho Ahn, Mattan Erez, and William J. Dally. 2005. Scatter-Add in Data Parallel Architectures. In Proceedings of the IEEE 11th International Symposium on High-Performance Computer Architecture (HPCA). 132-142. https://doi.org/10. 1109/HPCA.2005.30

[3] Mohammad Alian and Nam Sung Kim. 2019. NetDIMM: Low-Latency NearMemory Network Interface Architecture. In Proceedings of the 52nd Annual IEEE/ACM International Symposium on Microarchitecture (MICRO). Association for Computing Machinery, New York, NY, USA, 699-711. https://doi.org/10. $1145 / 3352460.3358278$

[4] Mohammad Alian, Seung Won Min, Hadi Asgharimoghaddam, Ashutosh Dhar Dong Kai Wang, Thomas Roewer, Adam McPadden, Oliver O'Halloran, Deming Chen, Jinjun Xiong, Daehoon Kim, Wen-mei Hwu, and Nam Sung Kim. 2018. Application-Transparent Near-Memory Processing Architecture with Memory Channel Network. In Proceedings of the 51st Annual IEEE/ACM International Symposium on Microarchitecture (MICRO). IEEE Press, 802-814. https://doi.org/ 10.1109/MICRO.2018.00070

[5] Bahar Asgari, Ramyad Hadidi, Jiashen Cao, Da Eun Shim, Sung-Kyu Lim, and Hyesoon Kim. 2021. FAFNIR: Accelerating Sparse Gathering by Using Efficient Near-Memory Intelligent Reduction. In Proceedings of the IEEE 27th International Symposium on High-Performance Computer Architecture (HPCA). 908-920. https: //doi.org/10.1109/HPCA51647.2021.00080

[6] Hadi Asghari-Moghaddam, Young Hoon Son, Jung Ho Ahn, and Nam Sung Kim 2016. Chameleon: Versatile and Practical Near-DRAM Acceleration Architecture for Large Memory Systems. In Proceedings of the 49th Annual IEEE/ACM International Symposium on Microarchitecture (MICRO). IEEE Press, Article 50, 13 pages.

[7] Sanguhn Cha, Seongil O, Hyunsung Shin, Sangjoon Hwang, Kwangil Park, Seong Jin Jang, Joo Sun Choi, Gyo Young Jin, Young Hoon Son, Hyunyoon Cho, Jung Ho Ahn, and Nam Sung Kim. 2017. Defect Analysis and Cost-Effective Resilience Architecture for Future DRAM Devices. In Proceedings of the IEEE 23rd International Symposium on High Performance Computer Architecture (HPCA). 61-72. https://doi.org/10.1109/HPCA.2017.30

[8] Paul Covington, Jay Adams, and Emre Sargin. 2016. Deep Neural Networks for YouTube Recommendations. In Proceedings of the 10th ACM Conference on Recommender Systems (RecSys). Association for Computing Machinery, New York, NY, USA, 191-198. https://doi.org/10.1145/2959100.2959190

[9] CriteoLabs. 2014. Kaggle Display Advertising Challenge Dataset. http://labs. criteo.com/2014/02/download-kaggle-display-advertising-challenge-dataset/

[10] William J. Dally and Brian P. Towles. 2004. Principles and Practices of Interconnection Networks. Morgan Kaufmann Publishers Inc.

[11] Fabrice Devaux. 2019. The true Processing In Memory accelerator. In 2019 IEEE Hot Chips 31 Symposium (HCS). 1-24. https://doi.org/10.1109/HOTCHIPS.2019. 8875680

[12] Mario Drumond, Alexandros Daglis, Nooshin Mirzadeh, Dmitrii Ustiugov, Javier Picorel, Babak Falsafi, Boris Grot, and Dionisios Pnevmatikatos. 2017. The Mondrian Data Engine. In Proceedings of the ACM/IEEE 44th Annual International Symposium on Computer Architecture (ISCA). Association for Computing Machinery, New York, NY, USA, 639-651. https://doi.org/10.1145/3079856.3080233

[13] Assaf Eisenman, Maxim Naumov, Darryl Gardner, Misha Smelyanskiy, Sergey Pupyrev, Kim Hazelwood, Asaf Cidon, and Sachin Katti. 2019. Bandana: Using Non-volatile Memory for Storing Deep Learning Models. In Proceedings of Machine Learning and Systems.

[14] Amin Farmahini-Farahani, Jung Ho Ahn, Katherine Morrow, and Nam Sung Kim. 2015. NDA: Near-DRAM Acceleration Architecture Leveraging Commodity DRAM Devices and Standard Memory Modules. In Proceedings of the IEEE 21st International Symposium on High Performance Computer Architecture (HPCA) 283-295. https://doi.org/10.1109/HPCA.2015.7056040

[15] Ivan Fernandez, Ricardo Quislant, Christina Giannoula, Mohammed Alser, Juan Gómez-Luna, Eladio Gutiérrez, Oscar Plata, and Onur Mutlu. 2020. NATSA: A Near-Data Processing Accelerator for Time Series Analysis. In Proceedings of the International Conference on Computer Design (ICCD).

[16] Mingyu Gao, Jing Pu, Xuan Yang, Mark Horowitz, and Christos Kozyrakis. 2017. TETRIS: Scalable and Efficient Neural Network Acceleration with 3D Memory. In Proceedings of the 22nd International Conference on Architectural Support for Programming Languages and Operating Systems (ASPLOS). Association for Computing Machinery, New York, NY, USA, 751-764. https://doi.org/10.1145/3037697. 3037702

[17] Peng Gu, Xinfeng Xie, Yufei Ding, Guoyang Chen, Weifeng Zhang, Dimin Niu, and Yuan Xie. 2020. iPIM: Programmable In-Memory Image Processing Accelerator Using Near-Bank Architecture. In Proceedings of the ACM/IEEE 47th Annual International Symposium on Computer Architecture (ISCA). IEEE Press, 804-817. https://doi.org/10.1109/ISCA45697.2020.00071

[18] Udit Gupta, Samuel Hsia, Vikram Saraph, Xiaodong Wang, Brandon Reagen, Gu-Yeon Wei, Hsien-Hsin S. Lee, David Brooks, and Carole-Jean Wu. 2020 DeepRecSys: A System for Optimizing End-to-End at-Scale Neural Recommendation Inference. In Proceedings of the ACM/IEEE 47th Annual International
Symposium on Computer Architecture (ISCA). IEEE Press, 982-995. https: //doi.org/10.1109/ISCA45697.2020.00084

[19] Udit Gupta, Samuel Hsia, Jeff Zhang, Mark Wilkening, Javin Pombra, HsienHsin S. Lee, Gu-Yeon Wei, Carole-Jean Wu, and David Brooks. 2021. RecPipe: Co-designing Models and Hardware to Jointly Optimize Recommendation Quality and Performance. (2021). arXiv:arXiv:2105.08820

[20] Udit Gupta, Carole-Jean Wu, Xiaodong Wang, Maxim Naumov, Brandon Reagen, David Brooks, Bradford Cottel, Kim Hazelwood, Mark Hempstead, Bill Jia, HsienHsin Lee, Andrey Malevich, Dheevatsa Mudigere, Mikhail Smelyanskiy, Liang Xiong, and Xuan Zhang. 2020. The Architectural Implications of Facebook's DNNBased Personalized Recommendation. In Proceedings of the IEEE 26th International Symposium on High Performance Computer Architecture (HPCA). 488-501. https: //doi.org/10.1109/HPCA47549.2020.00047

[21] Wenqin Huangfu, Xueqi Li, Shuangchen Li, Xing Hu, Peng Gu, and Yuan Xie. 2019. MEDAL: Scalable DIMM Based Near Data Processing Accelerator for DNA Seeding Algorithm. In Proceedings of the 52nd Annual IEEE/ACM International Symposium on Microarchitecture (MICRO). Association for Computing Machinery, New York, NY, USA, 587-599. https://doi.org/10.1145/3352460.3358329

[22] Ranggi Hwang, Taehun Kim, Youngeun Kwon, and Minsoo Rhu. 2020. Centaur: A Chiplet-Based, Hybrid Sparse-Dense Accelerator for Personalized Recommendations. In Proceedings of the ACM/IEEE 47th Annual International Symposium on Computer Architecture (ISCA). IEEE Press, 968-981. https://doi.org/10.1109/ ISCA45697.2020.00083

[23] Bongjoon Hyun, Youngeun Kwon, Yujeong Choi, John Kim, and Minsoo Rhu. 2020. NeuMMU: Architectural Support for Efficient Address Translations in Neural Processing Units. In Proceedings of the 25th International Conference on Architectural Support for Programming Languages and Operating Systems (ASPLOS). Association for Computing Machinery, New York, NY, USA, 1109-1124. https://doi.org/10.1145/3373376.3378494

[24] JEDEC. 2017. DDR4 SDRAM Standard.

[25] JEDEC. 2019. DDR4 Registering Clock Driver

[26] JEDEC. 2020. DDR5 SDRAM Standard.

[27] Norman P. Jouppi, Andrew B. Kahng, Naveen Muralimanohar, and Vaishnav Srinivas. 2012. CACTI-IO: CACTI With OFF-chip Power-Area-Timing Models. In Proceedings of the International Conference on Computer-Aided Design (ICCAD). Association for Computing Machinery, New York, NY, USA, 294-301. https: //doi.org/10.1145/2429384.2429446

[28] Dhiraj Kalamkar, Evangelos Georganas, Sudarshan Srinivasan, Jianping Chen, Mikhail Shiryaev, and Alexander Heinecke. 2020. Optimizing Deep Learning Recommender Systems Training on CPU Cluster Architectures. In International Conference for High Performance Computing, Networking, Storage and Analysis (SC). 1-15. https://doi.org/10.1109/SC41405.2020.00047

[29] Liu Ke, Udit Gupta, Benjamin Youngjae Cho, David Brooks, Vikas Chandra, Utku Diril, Amin Firoozshahian, Kim Hazelwood, Bill Jia, Hsien-Hsin S. Lee, Meng Li, Bert Maher, Dheevatsa Mudigere, Maxim Naumov, Martin Schatz, Mikhail Smelyanskiy, Xiaodong Wang, Brandon Reagen, Carole-Jean Wu, Mark Hempstead, and Xuan Zhang. 2020. RecNMP: Accelerating Personalized Recommendation with Near-Memory Processing. In Proceedings of the ACM/IEEE 47th Annual International Symposium on Computer Architecture (ISCA). IEEE Press, 790-803. https://doi.org/10.1109/ISCA45697.2020.00070

[30] Liu Ke, Xuan Zhang, Jinin So, Jong-Geon Lee, Shin-Haeng Kang, Sukhan Lee, Songyi Han, Yeongon Cho, Jin Hyun Kim, Yongsuk Kwon, Kyungsoo Kim, Jin Jung, Ilkwon Yun, Sung Joo Park, Hyunsun Park, Joonho Song, Jeonghyeon Cho, Kyomin Sohn, Nam Sung Kim, and Hsien-Hsin Sean Lee. 2021. Near-Memory Processing in Action: Accelerating Personalized Recommendation with AxDIMM. IEEE Micro 01 (July 2021), 1-1. https://doi.org/10.1109/MM.2021.3097700

[31] Byeongho Kim, Jaehyun Park, Eojin Lee, Minsoo Rhu, and Jung Ho Ahn. 2021. TRiM: Tensor Reduction in Memory. IEEE Computer Architecture Letters 20, 1 (Jan. 2021), 5-8. https://doi.org/10.1109/LCA.2020.3042805

[32] Duckhwan Kim, Jaeha Kung, Sek Chai, Sudhakar Yalamanchili, and Saibal Mukhopadhyay. 2016. Neurocube: A Programmable Digital Neuromorphic Architecture with High-Density 3D Memory. In Proceedings of the ACM/IEEE 43rd International Symposium on Computer Architecture (ISCA). IEEE Press, 380-392. https://doi.org/10.1109/ISCA.2016.41

[33] Dongkyun Kim, Minsu Park, Sungchun Jang, Jun-Yong Song, Hankyu Chi, Geunho Choi, Sunmyung Choi, Jaeil Kim, Changhyun Kim, Kyungwhan Kim, Kibong Koo, Seonghwi Song, Yongmi Kim, Dong Uk Lee, Jaein Lee, Daesuk Kim, Kihun Kwon, Minsik Han, Byeongchan Choi, Hongjung Kim, Sanghyun Ku, Yeonuk Kim, Jongsam Kim, Sanghui Kim, Youngsuk Seo, Seungwook Oh, Dain Im, Haksong Kim, Jonghyuck Choi, Jinil Chung, Changhyun Lee, Yongsung Lee, Joo-Hwan Cho, Junhyun Chun, and Jonghoon Oh. 2019. 23.2 A 1.1V 1ynm 6.4Gb/s/pin $16 \mathrm{~Gb}$ DDR5 SDRAM with a Phase-Rotator-Based DLL, High-Speed SerDes and RX/TX Equalization Scheme. In 2019 IEEE International Solid-State Circuits Conference (ISSCC). 380-382. https://doi.org/10.1109/ISSCC.2019.8662320

[34] Yoongu Kim, Weikun Yang, and Onur Mutlu. 2016. Ramulator: A Fast and Extensible DRAM Simulator. IEEE Computer Architecture Letters 15, 1 (Jan. 2016), 45-49. https://doi.org/10.1109/LCA.2015.2414456 
[35] Youngeun Kwon, Yunjae Lee, and Minsoo Rhu. 2019. TensorDIMM: A Practical Near-Memory Processing Architecture for Embeddings and Tensor Operations in Deep Learning. In Proceedings of the 52nd Annual IEEE/ACM International Symposium on Microarchitecture (MICRO). Association for Computing Machinery, New York, NY, USA, 740-753. https://doi.org/10.1145/3352460.3358284

[36] Youngeun Kwon, Yunjae Lee, and Minsoo Rhu. 2021. Tensor Casting: CoDesigning Algorithm-Architecture for Personalized Recommendation Training. In Proceedings of the IEEE 27th International Symposium on High-Performance Computer Architecture (HPCA). IEEE Computer Society, Los Alamitos, CA, USA, 235-248. https://doi.org/10.1109/HPCA51647.2021.00029

[37] Young-Cheon Kwon, Suk Han Lee, Jaehoon Lee, Sang-Hyuk Kwon, Je Min Ryu, Jong-Pil Son, Seongil O, Hak-Soo Yu, Haesuk Lee, Soo Young Kim, Youngmin Cho, Jin Guk Kim, Jongyoon Choi, Hyun-Sung Shin, Jin Kim, BengSeng Phuah, HyoungMin Kim, Myeong Jun Song, Ahn Choi, Daeho Kim, SooYoung Kim, EunBong Kim, David Wang, Shinhaeng Kang, Yuhwan Ro, Seungwoo Seo, JoonHo Song, Jaeyoun Youn, Kyomin Sohn, and Nam Sung Kim. 2021. 25.4 A 20nm 6GB Function-In-Memory DRAM, Based on HBM2 with a 1.2TFLOPS Programmable Computing Unit Using Bank-Level Parallelism, for Machine Learning Applications. In 2021 IEEE International Solid-State Circuits Conference (ISSCC), Vol. 64 350-352. https://doi.org/10.1109/ISSCC42613.2021.9365862

[38] Sukhan Lee, Shin-haeng Kang, Jaehoon Lee, Hyeonsu Kim, Eojin Lee, Seungwoo Seo, Hosang Yoon, Seungwon Lee, Kyounghwan Lim, Hyunsung Shin, Jinhyun Kim, Seongil O, Anand Iyer, David Wang, Kyomin Sohn, and Nam Sung Kim. 2021. Hardware Architecture and Software Stack for PIM Based on Commercial DRAM Technology : Industrial Product. In 2021 ACM/IEEE 48th Annual International Symposium on Computer Architecture (ISCA). IEEE Computer Society, Los Alamitos, CA, USA, 43-56. https://doi.org/10.1109/ISCA52012.2021.00013

[39] Jie Li, Xi Wang, Antonino Tumeo, Brody Williams, John D. Leidel, and Yong Chen. 2019. PIMS: A Lightweight Processing-in-Memory Accelerator for Stencil Computations. In Proceedings of the International Symposium on Memory Systems (MEMSYS). Association for Computing Machinery, New York, NY, USA, 41-52. https://doi.org/10.1145/3357526.3357550

[40] Shuangchen Li, Alvin Oliver Glova, Xing Hu, Peng Gu, Dimin Niu, Krishna T. Malladi, Hongzhong Zheng, Bob Brennan, and Yuan Xie. 2018. SCOPE: A Stochastic Computing Engine for DRAM-Based In-Situ Accelerator. In Proceedings of the 51st Annual IEEE/ACM International Symposium on Microarchitecture (MICRO) IEEE Press, 696-709. https://doi.org/10.1109/MICRO.2018.00062

[41] Shuangchen Li, Dimin Niu, Krishna T. Malladi, Hongzhong Zheng, Bob Brennan, and Yuan Xie. 2017. DRISA: A DRAM-Based Reconfigurable In-Situ Accelerator In Proceedings of the 50th Annual IEEE/ACM International Symposium on Microarchitecture (MICRO). Association for Computing Machinery, New York, NY, USA, 288-301. https://doi.org/10.1145/3123939.3123977

[42] Jiawen Liu, Hengyu Zhao, Matheus Almeida Ogleari, Dong Li, and Jishen Zhao. 2018. Processing-in-Memory for Energy-Efficient Neural Network Training: A Heterogeneous Approach. In Proceedings of the 51st Annual IEEE/ACM International Symposium on Microarchitecture (MICRO). IEEE Press, 655-668 https://doi.org/10.1109/MICRO.2018.00059

[43] Shih-Lien Lu, Ying-Chen Lin, and Chia-Lin Yang. 2015. Improving DRAM Latency with Dynamic Asymmetric Subarray. In Proceedings of the 48th Annual IEEE/ACM International Symposium on Microarchitecture (MICRO). As sociation for Computing Machinery, New York, NY, USA, 255-266. https: //doi.org/10.1145/2830772.2830827

[44] David Luebke. 2008. CUDA: Scalable Parallel Programming for High-Performance Scientific Computing. In 2008 5th IEEE International Symposium on Biomedical Imaging: From Nano to Macro (ISBI). 836-838. https://doi.org/10.1109/ISBI.2008. 4541126

[45] Micron. 2017. Calculating Memory Power for DDR4 SDRAM

[46] Maxim Naumov, Dheevatsa Mudigere, Hao-Jun Shi, Jianyu Huang, Narayanan Sundaraman, Jongsoo Park, Xiaodong Wang, Udit Gupta, Carole-Jean Wu, Alis son Azzolini, Dmytro Dzhulgakov, Andrey Mallevich, Ilia Cherniavskii, Yinghai Lu, Raghuraman Krishnamoorthi, Ansha Yu, Volodymyr Kondratenko, Stephanie Pereira, Xianjie Chen, and Misha Smelyanskiy. 2019. Deep Learning Recommendation Model for Personalization and Recommendation Systems. (2019) arXiv:arXiv:1906.00091

[47] Rajesh Nishtala, Hans Fugal, Steven Grimm, Marc Kwiatkowski, Herman Lee, Harry Li, Ryan McElroy, Mike Paleczny, Daniel Peek, Paul Saab, David Stafford, Tony Tung, and Venkateshwaran Venkataramani. 2013. Scaling Memcache at Facebook. In 10th USENIX Symposium on Networked Systems Design and Implementation (NSDI). USENIX Association, Lombard, IL, 385-398. https //www.usenix.org/conference/nsdi13/technical-sessions/presentation/nishtala

[48] Seongil O, Sanghyuk Kwon, Young Hoon Son, Yujin Park, and Jung Ho Ahn 2015. CIDR: A Cache Inspired Area-Efficient DRAM Resilience Architecture against Permanent Faults. IEEE Computer Architecture Letters 14, 1 (2015), 17-20.
https://doi.org/10.1109/LCA.2014.2324894

[49] Seongil O, Young Hoon Son, Nam Sung Kim, and Jung Ho Ahn. 2014. Row-Buffer Decoupling: A Case for Low-Latency DRAM Microarchitecture. In Proceedings of the ACM/IEEE 41st Annual International Symposium on Computer Architecuture (ISCA). IEEE Press, 337-348.

[50] Mike O'Connor, Niladrish Chatterjee, Donghyuk Lee, John Wilson, Aditya Agrawal, Stephen W. Keckler, and William J. Dally. 2017. Fine-Grained DRAM: Energy-Efficient DRAM for Extreme Bandwidth Systems. In Proceedings of the 50th Annual IEEE/ACM International Symposium on Microarchitecture (MICRO). Association for Computing Machinery, New York, NY, USA, 41-54. https: //doi.org/10.1145/3123939.3124545

[51] Tae-Young Oh, Hoeju Chung, Jun-Young Park, Ki-Won Lee, Seunghoon Oh, Su-Yeon Doo, Hyoung-Joo Kim, ChangYong Lee, Hye-Ran Kim, Jong-Ho Lee, Jin-Il Lee, Kyung-Soo Ha, YoungRyeol Choi, Young-Chul Cho, Yong-Cheol Bae, Taeseong Jang, Chulsung Park, Kwangil Park, SeongJin Jang, and Joo Sun Choi. 2015. A $3.2 \mathrm{Gbps} /$ pin 8 Gbit 1.0 V LPDDR4 SDRAM With Integrated ECC Engine for Sub-1 V DRAM Core Operation. IEEE fournal of Solid-State Circuits 50, 1 (2015), 178-190. https://doi.org/10.1109/JSSC.2014.2353799

[52] Minesh Patel, Jeremie Kim, Taha-Michael Shahroodi, Hasan Hassan, and Onur Mutlu. 2020. Bit-Exact ECC Recovery (BEER): Determining DRAM On-Die ECC Functions by Exploiting DRAM Data Retention Characteristics. In Proceedings of the 53rd Annual IEEE/ACM International Symposium on Microarchitecture (MICRO).

[53] William W. Peterson and Edward J. Weldon. 1972. Error-correcting codes. MIT press.

[54] Vijay Janapa Reddi, Christine Cheng, David Kanter, Peter Mattson, Guenther Schmuelling, Carole-Jean Wu, Brian Anderson, Maximilien Breughe, Mark Charlebois, William Chou, Ramesh Chukka, Cody Coleman, Sam Davis, Pan Deng, Greg Diamos, Jared Duke, Dave Fick, J. Scott Gardner, Itay Hubara, Sachin Idgunji, Thomas B. Jablin, Jeff Jiao, Tom St. John, Pankaj Kanwar, David Lee, Jeffery Liao, Anton Lokhmotov, Francisco Massa, Peng Meng, Paulius Micikevicius, Colin Osborne, Gennady Pekhimenko, Arun Tejusve Raghunath Rajan, Dilip Sequeira, Ashish Sirasao, Fei Sun, Hanlin Tang, Michael Thomson, Frank Wei, Ephrem Wu, Lingjie Xu, Koichi Yamada, Bing Yu, George Yuan, Aaron Zhong, Peizhao Zhang, and Yuchen Zhou. 2020. MLPerf Inference Benchmark. In Proceedings of the ACM/IEEE 47th Annual International Symposium on Computer Architecture (ISCA). IEEE Press, 446-459. https://doi.org/10.1109/ISCA45697.2020.00045

[55] Samsung Electronics. 2017. 8Gb B-die DDR4 SDRAM. https: //www.samsung.com/semiconductor/global.semi/file/resource/2017/11/ 8G_B_DDR4_Samsung_Spec_Rev2_1_Feb_17-0.pdf

[56] Hao-Jun Michael Shi, Dheevatsa Mudigere, Maxim Naumov, and Jiyan Yang. 2020. Compositional Embeddings Using Complementary Partitions for MemoryEfficient Recommendation Systems. (2020), 165-175. https://doi.org/10.1145/ 3394486.3403059

[57] Hyunsung Shin, Dongyoung Kim, Eunhyeok Park, Sungho Park, Yongsik Park, and Sungjoo Yoo. 2018. McDRAM: Low Latency and Energy-Efficient Matrix Computations in DRAM. IEEE Transactions on Computer-Aided Design of Integrated Circuits and Systems 37, 11 (2018), 2613-2622. https://doi.org/10.1109/ TCAD.2018.2857044

[58] Young Hoon Son, Sukhan Lee, Seongil O, Sanghyuk Kwon, Nam Sung Kim, and Jung Ho Ahn. 2015. CiDRA: A Cache-inspired DRAM Resilience Architecture. In Proceedings of the IEEE 21st International Symposium on High Performance Computer Architecture (HPCA). 502-513. https://doi.org/10.1109/HPCA.2015. 7056058

[59] Young Hoon Son, Seongil O, Yuhwan Ro, Jae W. Lee, and Jung Ho Ahn. 2013. Reducing Memory Access Latency with Asymmetric DRAM Bank Organizations. In Proceedings of the ACM/IEEE 40th Annual International Symposium on Computer Architecture (ISCA). Association for Computing Machinery, New York, NY, USA, 380-391. https://doi.org/10.1145/2485922.2485955

[60] Mengdi Wang, Chen Meng, Guoping Long, Chuan Wu, Jun Yang, Wei Lin, and Yangqing Jia. 2019. Characterizing Deep Learning Training Workloads on AlibabaPAI. 189-202. https://doi.org/10.1109/IISWC47752.2019.9042047

[61] Mark Wilkening, Udit Gupta, Samuel Hsia, Caroline Trippel, Carole-Jean Wu, David Brooks, and Gu-Yeon Wei. 2021. RecSSD: Near Data Processing for Solid State Drive Based Recommendation Inference. In Proceedings of the 26th International Conference on Architectural Support for Programming Languages and Operating Systems (ASPLOS). Association for Computing Machinery, New York, NY, USA, 717-729. https://doi.org/10.1145/3445814.3446763

[62] Carole-Jean Wu, Robin Burke, Ed Chi, Joseph Konstan, Julian McAuley, Yves Raimond, and Hao Zhang. 2020. Developing a Recommendation Benchmark for MLPerf Training and Inference. (2020). arXiv:arXiv:2003.07336

[63] Jie Amy Yang, Jianyu Huang, Jongsoo Park, Ping Tak Peter Tang, and Andrew Tulloch. 2020. Mixed-Precision Embedding Using a Cache. (2020). arXiv:arXiv:2010.11305 